Alll02 66013l

NATL INST OF STANDARDS \& TECH R.I.C.

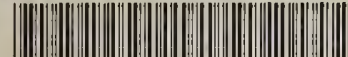

A11102660131

Frank, Danlel E/Ballistlc tests of used QC100.U56 NO.86-3444 1986 V19 C.1 NBS-P

IVDOIn OO-J444

\title{
Ballistic Tests of Used Soft Body Armor
}

Daniel E. Frank

U.S. DEPARTMENT OF COMMERCE

National Bureau of Standards

National Engineering Laboratory

Law Enforcement Standards Laboratory

Gaithersburg, MD 20899

August 1986

Issued September 1986

Prepared for

\section{National Institute of Justice}

- QC— partment of Justice

100 Iton, DC 20531

.456

86-3444

1986 



\section{BALLISTIC TESTS OF USED SOFT BODY ARMOR}

Daniel E. Frank

U.S. DEPARTMENT OF COMMERCE

National Bureau of Standards

National Engineering Laboratory

Law Enforcement Standards Laboratory

Gaithersburg, MD 20899

August 1986

Issued September 1986

Prepared for

National Institute of Justice

U.S. Department of Justice

Washington, DC 20531

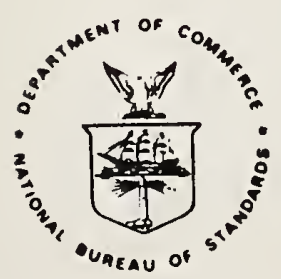

U.S. DEPARTMENT OF COMMERCE, Malcolm Baldrige, Secretary NATIONAL BUREAU OF STANDARDS. Ernest Ambler, Director 



\section{ACKNOWLEDGMENTS}

This report was prepared by the Law Enforcement Standards Laboratory of the National Bureau of Standards under the direction of Lawrence K. Eliason, Chief of LESL. The National Institute of Justice Technology Assessment Program (TAP) Information Center awarded the contract and monitored the ballistic testing described in this report, which was conducted by H. P. White Laboratory, Inc., Street, MD 21154. Mr. Brad Stimson, National Research Council of Canada, Public Safety Project office, participated in the test design and witnessing of the actual testing. The contributions of Marc Caplan, TAP Information Center are likewise acknowledged. The research was sponsored by the National Institute of Justice, Lester D. Shubin, Standards Program Manager. The technical effort to develop this report was conducted under Interagency Agreement LEAA-J-IAA-021-3, Project No. 7101. 

FOREWORD

The Law Enforcement Standards Laboratory (LESL) of the National Bureau of Standards (NBS) furnishes technical support to the National Institute of Justice (NIJ) program to strengthen law enforcement and criminal justice in the United States. LESL's function is to conduct research that will assist law enforcement and criminal justice agencies in the selection and procurement of quality equipment.

LESL is: (1) subjecting existing equipment to laboratory testing and evaluation and (2) conducting research leading to the development of several series of documents, including national voluntary equipment standards, user guides and technical reports.

This document presents the results of a joint NIJ and National Research Council (NRC) of Canada effort to evaluate the effect of age upon the ballistic-resistant capabilities of police body armor. The testing program was administered by the NIJ Technology Assessment Program Information Center assisted by LESL in support of NRC and NIJ.

Technical comments and suggestions concerning this document are invited from all interested parties. They may be addressed to the author or to the Law Enforcement Standards Laboratory, National Bureau of Standards, Gaithersburg, MD 20899.

Lawrence K. Eliason, Chief

Law Enforcement Standards Laboratory 

Foreword . . . . . . . . . . . . . . . . . . . . . . . . . . iii

1. Introduction . . . . . . . . . . . . . . . . . . . . . . . . 1

2. Background . . . . . . . . . . . . . . . . . . . . . . . . 2

3. Ballistic Resistance Test Methods. . . . . . . . . . . . . . . . . . . . 4

4. Test Specimens . . . . . . . . . . . . . . . . . . . . . . 6

5. Test Results . . . . . . . . . . . . . . . . . . . . . . . . 9

6. Discussion . . . . . . . . . . . . . . . . . . . . . . 16

7. Conclusions . . . . . . . . . . . . . . . . . . . . 19

8. References . . . . . . . . . . . . . . . . . . . . . . . . 20

Appendix A- $\mathrm{V}_{50}$ Ballistic Limit Test Data, Seven Layer Kevlar Soft Body Armor,

38 Caliber, 158 Grain, Lead Round Nose Projectile . . . . . . . . . . 21

Appendix B- $-V_{50}$ Ballistic Limit Test Data, Seven Layer Kevlar Soft Body Armor,

22 Caliber, 40 Grain, Lead Round Nose Projectile . . . . . . . . . . 28 


\begin{tabular}{|c|c|c|c|c|c|}
\hline A & ampere & $\mathrm{H}$ & henry & $\mathrm{nm}$ & nanometer \\
\hline ac & alternating current & $\mathrm{h}$ & hour & No. & number \\
\hline AM & amplitude modulation & hf & high frequency & o.d. & outside diameter \\
\hline cd & candela & $\mathrm{Hz}$ & hertz $(\mathrm{c} / \mathrm{s})$ & $\Omega$ & ohm \\
\hline $\mathrm{cm}$ & centimeter & i.d. & inside diameter & p. & page \\
\hline $\mathrm{CP}$ & chemically pure & in & inch & $\mathrm{Pa}$ & pascal \\
\hline $\mathrm{c} / \mathrm{s}$ & cycle per second & ir & infrared & pe & probable error \\
\hline d & day & $\mathbf{J}$ & joule & pp. & pages \\
\hline $\mathrm{dB}$ & decibel & L & lambert & ppm & part per million \\
\hline $\mathrm{dc}$ & direct current & $\mathrm{L}$ & liter & qt & quart \\
\hline${ }^{\circ} \mathrm{C}$ & degree Celsius & $\mathrm{lb}$ & pound & $\mathrm{rad}$ & radian \\
\hline${ }^{\circ} \mathrm{F}$ & degree Fahrenheit & lbf & pound-force & rf & radio frequency \\
\hline diam & diameter & $\mathrm{lbf} \cdot \mathrm{in}$ & pound-force inch & rh & relative humidity \\
\hline emf & electromotive force & $\operatorname{lm}$ & lumen & $\mathbf{s}$ & second \\
\hline eq & equation & $\ln$ & logarithm (natural) & $\mathrm{SD}$ & standard deviation \\
\hline$F$ & farad & $\log$ & logarithm (common) & sec. & section \\
\hline fc & footcandle & $M$ & molar & SWR & standing wave radio \\
\hline fig. & figure & $\mathrm{m}$ & meter & uhf & ultrahigh frequency \\
\hline FM & frequency modulation & $\min$ & minute & uv & ultraviolet \\
\hline $\mathrm{ft}$ & foot & $\mathrm{mm}$ & millimeter & V & volt \\
\hline $\mathrm{ft} / \mathrm{s}$ & foot per second & $\mathrm{mph}$ & mile per hour & vhf & very high frequency \\
\hline$g$ & acceleration & $\mathrm{m} / \mathrm{s}$ & meter per second & W & watt \\
\hline g & gram & $\mathrm{N}$ & newton & $\lambda$ & wavelength \\
\hline gr & grain & $\mathrm{N} \cdot \mathrm{m}$ & newton meter & $w t$ & weight \\
\hline
\end{tabular}

area $=$ unit $^{2}$ (e.g., $\mathrm{ft}^{2}$, in $^{2}$, etc.); volume $=$ unit $^{3}$ (e.g., $\mathrm{ft}^{3}, \mathrm{~m}^{3}$, etc. $)$

\section{PREFIXES}

$\begin{array}{ll}\text { d } & \text { deci }\left(10^{-1}\right) \\ \text { c } & \text { centi }\left(10^{-2}\right) \\ \text { m } & \text { milli }\left(10^{-3}\right) \\ \mu & \text { micro }\left(10^{-6}\right) \\ \text { n } & \text { nano }\left(10^{-9}\right) \\ \text { p } & \text { pico }\left(10^{-12}\right)\end{array}$

$\begin{array}{ll}\text { da } & \text { deka }(10) \\ \text { h } & \text { hecto }\left(10^{2}\right) \\ \mathrm{k} & \text { kilo }\left(10^{3}\right) \\ \mathrm{M} & \text { mega }\left(10^{6}\right) \\ \mathrm{G} & \text { giga }\left(10^{9}\right) \\ \mathrm{T} & \text { tera }\left(10^{12}\right)\end{array}$

COMMON CONVERSIONS

(See ASTM E380)

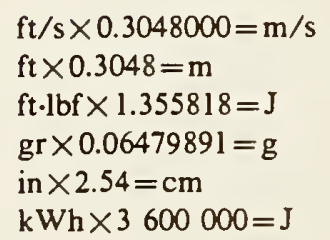

Temperature: $\left(T \cdot{ }_{F}-32\right) \times 5 / 9=T \cdot C$

Temperature: $(T \cdot \mathrm{c} \times 9 / 5)+32=T \cdot F$

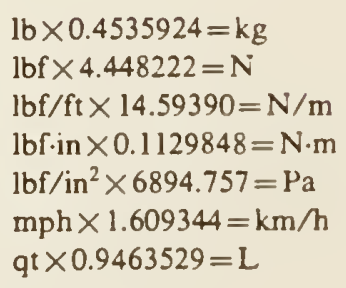




\section{Ballistic Tests of Used Soft Body Armor \\ Daniel E. Frank* \\ National Bureau of Standards \\ Gaithersburg, MD 20899}

A sample of 24 ballistic resistant undergarments (soft body armor) from a production lot of 1500 originally distributed to 15 police departments throughout the United States in 1975 for issue to officers as part of a Law Enforcement Assistance Administration demonstration project, was tested for $\mathrm{V}_{50}$ ballistic limit. The program was a joint effort of the U.S. Department of Justice National Institute of Justice and the National Research Council of Canada Public Safety Project office. Tests of ballistic limit were conducted on virgin armor that were never issued, and armor showing evidence of light, moderate, and heavy wear both dry and while wet. The results show that armor does not lose ballistic efficiency as a consequence of age.

Key words: ballistic limit; ballistic-resistant body armor; ballistic testing; body armor; Kevlar; soft body armor

1. INTRODUCTION ${ }^{1}$

Soft body armor suitable for routine full time use by police officers became available in quantity in the mid 1970's following development by the National Institute of Justice (NIJ), previously the National Institute of Law Enforcement and criminal Justice of the Law Enforcement Assistance Administration. In the interim, soft body armor manufactured from Kevlar ${ }^{2}$ aramid fiber fabric has gained widespread use. While

* Law Enforcement Standards Laboratory, National Engineering Laboratory.

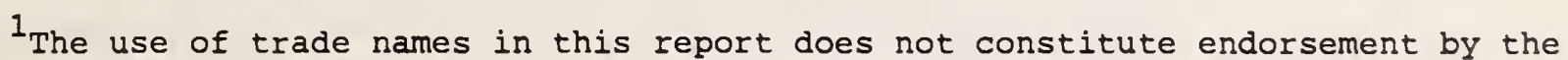
National Bureau of Standards, the U.S. Department of Justice, or any other government agency; nor does it imply that a product is necessarily best suited for the intended use.

${ }^{2}$ Registered trade name of E. I. Du Pont de Nemours \& Co., Inc. 
exact statistics are not available it is estimated that more than 50 percent of the nation's police have been issued body armor, or have purchased it themselves.

Many police departments are currently continuing to use armor that was purchased prior to 1975. Although there has never been a reported incident of armor manufactured from Kevlar failing to protect an officer when assaulted with a weapon having a ballistic threat equal to or less than the rated protection of the armor, those departments with older armor are increasingly concerned with the effect of age and wear upon Kevlar fabric.

During the last year, the NIJ Technology Assessment Program (TAP) Information Center, and the National Research Council of Canada (NRC) Public Safety Project office have received numerous inquiries from police agencies questioning whether it is necessary to replace older existing armor to be sure that their officers are properly protected. In response to these questions, NIJ and NRC requested that the National Bureau of Standards Law Enforcement Standards Laboratory (LESL) collaborate with the TAP Information Center to conduct tests of soft body armor that had been in service for extended periods of time. The discussion that follows describes the testing program that was conducted and presents the results of this effort.

\section{BACKGROUND}

The NIJ, aware of the rapidly increasing number of officer fatalities through handgun assault during the late 1960's and early 1970's recognized that the physical properties of Kevlar held potential for ballistic resistance. Preliminary experiments demonstrated that Kevlar was highly efficient in ballistic resistance and NIJ launched an effort to develop soft body armor that was suitable for routine full time usc by police officers.

The objective of the development effort was to design armor that would protect officers from the most common handgun threats of that time, the 38 caliber bullet at a velocity of $850 \pm 50 \mathrm{ft} / \mathrm{s}$, and the 22 caliber bullet at a velocity of $1050 \pm 50 \mathrm{ft} / \mathrm{s}$. Experiments were conducted to determine the minimum number of layers of Kevlar required 
to provide the desired ballistic protection, and it was found that seven layers were suitable. Since Kevlar fabric was not available in commercial quantity at the time, NIJ awarded contracts to several weavers to produce large quantities of the fabric. Following this, NIJ awarded contracts to several manufacturers to produce ballistic-resistant undergarments in accordance with the NIJ design specification. A total of 3000 such garments of two designs were manufactured, together with 2000 additional garments of several other types of soft body armor.

In order to prove that the new armor was effective in protecting the officers from handgun assault, and that it was suitable for full time routine use throughout the United States, NIJ distributed the armor to 15 cities throughout the United States. The resulting field test verified that all of the objectives of the NIJ development effort had been met $[1]^{3}$, and body armor manufacturers began to actively market the new armor.

During the time that NIJ developed the new soft body armor, LESL developed a performance standard for body armor, which was promulgated by NIJ as a voluntary national standard in 1973. Since then, the NIJ standard, which has been revised twice to remain current with technology, has been widely used both domestically and internationally as the basis upon which body armor is purchased.

During the course of its development effort, NIJ was careful to document the details of the experimental effort. Thus, data were available concerning the ballistic-resistant characteristics of the original production lots that would enable valid conclusions concerning the effect, of age and wear, if any, upon the ballistic efficiency of those vests if samples could be obtained for laboratory tests.

The TAP Information Center contacted each of the 15 cities that had been given undergarments during the NIJ demonstration program and requested a search of property records to determine if any of the vests were still in their possession. In addition, NIJ examined its own property records and requested that other Federal agencies that purchased armor in parallel with the NIJ program do the same.

${ }^{3}$ Numbers in brackets refer to references in section 8 of this report. 
Five cities located armor from the original NIJ purchase, some still in actual use and some in inventory. Similarly, NIJ and two other Federal agencies were able to locate armor manufactured at that time, some of which was never worn.

\section{BALLISTIC RESISTANCE TEST METHODS}

The physical characteristics of Kevlar fiber, and the fabric woven from it, vary somewhat from lot to lot and even within a lot, as with any item of manufacture. As a consequence, when multiple layers of fabric are used to construct soft body armor the ballistic resistance of individual vests of the same design varies from one to another.

The NIJ standard for ballistic resistant police body armor establishes minimum performance requirements. To this end, body armor is tested by firing specific types of bullets against armor samples using closely controlled velocities. Armor that is not penetrated by the required test rounds and does not deform more than 1.73 in upon impact is considered to meet the requirements of the standard. Because soft body armor manufactured from fabric is known to lose ballistic efficiency when it is wet, and since officers do get wet, the armor model is tested both dry and while wet.

The NIJ standard for body armor can be used as the basis for tests to determine whether armor complies with the minimum performance requirements as specified, but the test results do not provide a knowledge of the ultimate ballistic protection that a given sample of body armor may provide. Frequently manufacturers will incorporate more layers of fabric than required for minimum performance to ensure that the armor will meet the ballistic requirements even if a given lot of fabric is slightly less ballistically efficient than normal.

In order to examine the relative ballistic performance of armor, rather than simply verifying minimum performance, it becomes necessary to use a different method of test. The armor industry has typically used the $\mathrm{v}_{50}$ ballistic limit as the means of comparing the ultimate performance of armor materials. 
$\mathrm{V}_{50}$ ballistic limit is the velocity at which a specific projectile (bullet) is expected to penetrate the armor half of the time.

The ballistic limit of armor is most frequently conducted using the procedures of MIL-STD-662D [2]. Essentially, the specified test projectile is fired at the armor over a range of impact velocities and the specimen examined after each impact to determine whether the projectile has penetrated the armor or not. While the standard permits different velocity ranges from the highest velocity test round to the lowest, a total velocity range of $125 \mathrm{ft} / \mathrm{s}$ is most commonly used for the test. In practice, the first projectile is fired at a velocity controlled so as to yield an impact velocity near that of the expected $v_{50}$. If the first projectile penetrates the velocity of the second test round is reduced and the impact point examined for penetration. Conversely, if the first projectile does not penetrate, the velocity of the second round is increased and the impact point examined for penetration. The objective is to fire a total of 10 projectiles at various velocities to obtain 10 impacts; five of which penetrate and five of which do not within an overall velocity range of $125 \mathrm{ft} / \mathrm{s}$. The $v_{50}$ ballistic limit is then calculated as the average velocity of the 10 test rounds.

The test results presented in this report were obtained using the procedures of MIL-STD-662D.

The NIJ standard for police body armor requires the evaluation of both the penetration resistance of body armor and the deformation of the armor caused by the bullet impact. The deformation, which is measured as the depth of the indentation in the clay backing material at the point of a nonpenetrating impact, is determined to ensure that an individual wearing the armor will be protected from blunt trauma.

Specifically, NILECJ-STD-0101.01, Ballistic Resistance of Police Body Armor [3] (the test method used to obtain some of the results presented in this report) requires that the deformation not exceed 1.73 in for Type I armor when impacted with a 40 grain lead round nose 22 caliber bullet at a velocity of $1050 \pm 50 \mathrm{ft} / \mathrm{s}$ and a $158 \mathrm{grain}$ lead round nose 38 caliber bullet at velocity of $850 \pm 50 \mathrm{ft} / \mathrm{s}$. 
The deformation measurements were included in the experimental design to determine whether deformation might be an early indicator of ballistic deterioration, should used armor exhibit a significant loss in ballistic efficiency.

\section{TEST SPECIMENS}

The 24 sets of body armor tested in this program were all of the same design as shown in figures $1 \mathrm{a}$ and $1 \mathrm{~b}$ and the vests were constructed of seven layers of 1000 denier, $31 \times 31$ plain weave Kevlar fabric, which was waterproofed with Zepel $\mathrm{D}^{2}$.
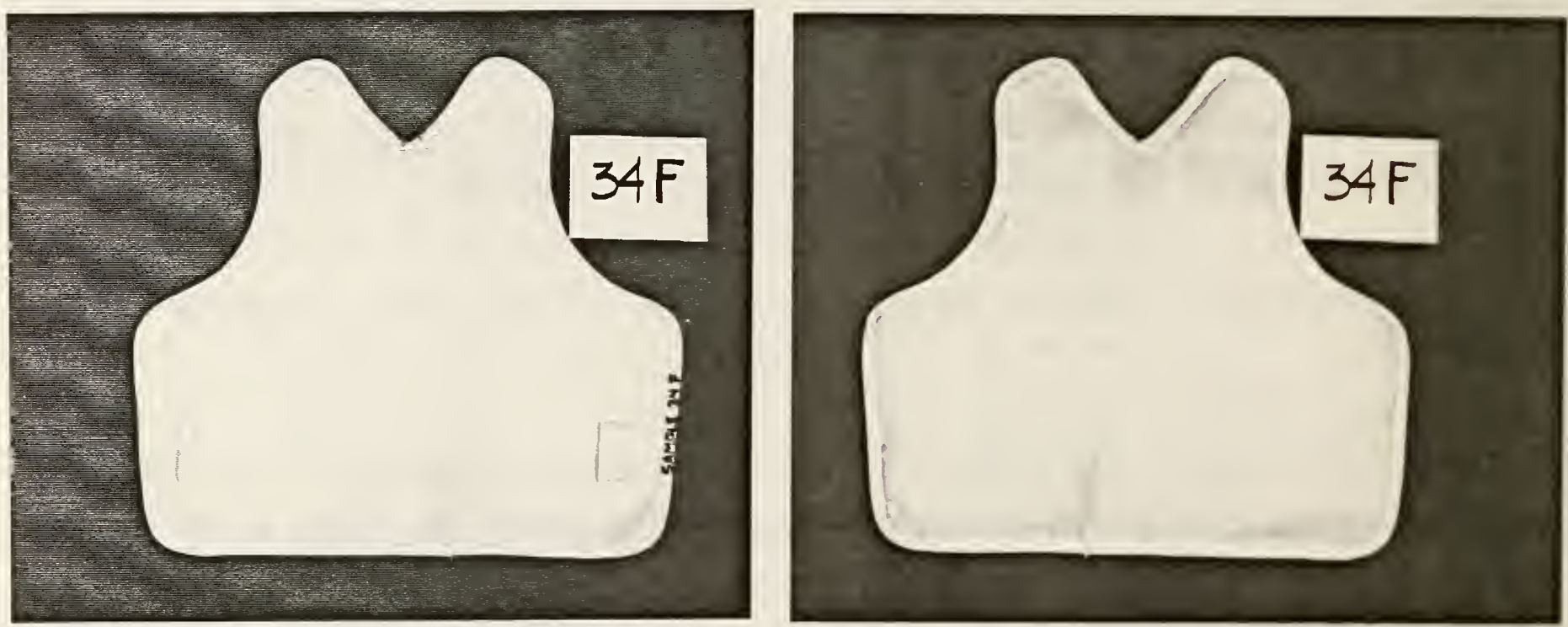

Figure 1a. Front of vest outside and inside. 

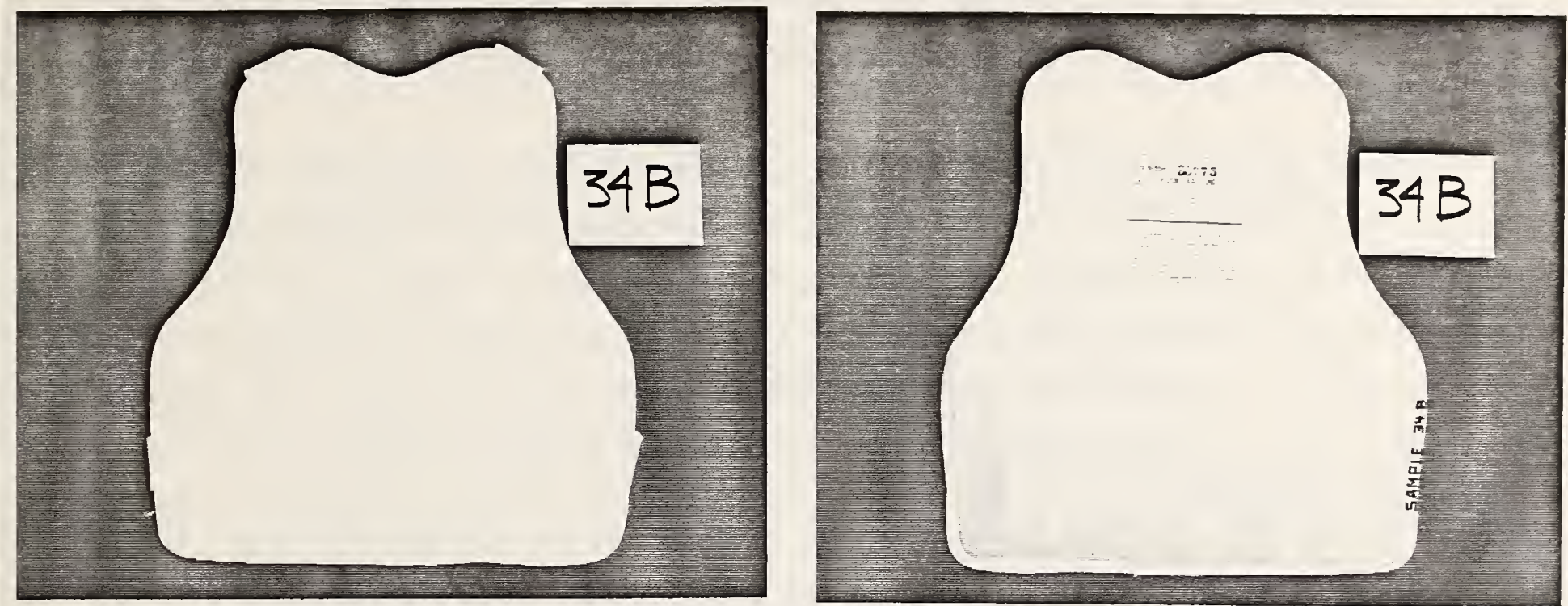

Figure 1b. Back of vest outside and inside.

During manufacture, the two outer layers of Kevlar fabric and the exterior cover were cut to the finished size. The five interior layers were cut slightly smaller than the outer layers. The interior Kevlar layers were first stitched together at a number of points near the edge of the fabric. The interior layers were then placed between the two outer Kevlar layers and the front cover and the assembly was completed by sewing bias tape around the entire edge attached to the outer Kevlar layers, which extended approximately one-half inch beyond the edge of the interior layers.

The back panel construction was identical to that of the front panel as described above; however, in addition, the back panel was reinforced by vertical stitching from top to bottom at intervals of approximately 4 in from one side to the other.

Each vest was clearly labeled "LEAA Prototype Protective Garment," which enabled verification that the garment was indeed from the original LEAA demonstration production lot.

While the property records of the five police departments were sufficiently accurate to locate the test specimens, it was generally not possible to obtain accurate information concerning the wear and maintenance details for the individual vests. This 
was a consequence of the fact that the individuals that were issued the vests were no longer on their respective forces and efforts to locate the officers were unsuccessful.

It was possible to obtain limited use information for only seven of the vests. Five of the vests $(15,17,18,20$, and 21) were obtained from a department with a very hot, moderately humid climate. These vests were issued upon receipt from LEAA in 1975 and were still in service when recalled for testing in April 1986--a service period well over 10 years. The department policy was one of voluntary use, however, the department believes that the officers that wore the vests did so full time except for the hottest months of the summer.

Vest 6 which came from a department with a generally hot, humid climate, was worn by three different officers. The first officer had the vest for over a year and one-half; however, its use was not known. The second officer wore it full time while on duty for approximately two years, wiping it with a damp sponge and soap and never machine washing it. The last officer wore it for a one-year period but the extent of wear is not known.

Vest 9 which came from a department with a very hot humid climate, was used by the first officer of issue for over three and one-half years. There is no information on the extent of use. The vest was subsequently issued to an auxiliary officer that wore it full time while on duty for a period of five years. However, this officer was only on duty a few days each month.

In the absence of detailed use information, it became necessary to rely upon visual inspection to estimate the extent of use. Eight of the 24 test specimens were unused. Representatives of NIJ, LESL, and the TAP Information Center examined each of the remaining 16 specimens that had been used. It was possible to separate the vests into three groups:

- Four exhibited light wear

- Four exhibited moderate wear

- Eight exhibited heavy wear 
Once the test specimens were classified in accordance with use (unused, light wear, moderate wear, heavy wear), they were randomly assigned to two test groups. One group was scheduled for ballistic test using 38 caliber ammunition, the other for tests using 22 caliber ammunition. Finally, half of the vests in each group were scheduled for ballistic tests while wet and the other half for ballistic tests while dry.

\section{TEST RESULTS}

The testing of used armor was conducted by the H. P. White Laboratory, Inc., Street, Maryland, during the period from May 22 to June 10, 1986. Representatives of the TAP Information Center, the National Research Council of Canada, and LESL witnessed all testing.

The test samples were mounted at a distance of $16.0 \mathrm{ft}$ from the test weapon on clay backing as specified by NILECJ-STD-0101.01 to produce a zero degree angle of obliquity. The first shots on each panel were to determine the back face signature (clay deformation) in accordance with NILECJ-STD-0101.01 after which the $v_{50}$ of the panel was determined. Light screens were positioned at 6.5 and $9.5 \mathrm{ft}$ which, in conjunction with an elapsed time counter (chronograph), were used to determine all bullet velocities at $8.0 \mathrm{ft}$.

In conducting ballistic limit tests of the 24 samples, the front and back panels of each vest were tested separately. Every effort was made to obtain 10 valid impacts for the determination of $v_{50}$ ballistic limit. However, there were a number of instances in which the shot placement was such that it was not possible to obtain 10 valid impacts and, in these cases, 8 shot $v_{50}$ ballistic limit data are reported. Similarly, there were instances in which the inspection of the vest at a later date revealed that an impact used to calculate a 10 shot $v_{50}$ ballistic limit was not a valid impact and the data was recalculated on the basis of an 8 shot $v_{50}$.

Table 1 summarizes the $v_{50}$ ballistic limit for each vest tested using 38 caliber bullets. Following completion of the tests, vest 22 was reinspected and found to contain eight layers of Kevlar, rather than the specified seven layers. Table 2 
presents the blunt trauma deformation measurements that were obtained using 38 caliber bullets.

Table 1. $\mathrm{V}_{50}$ ballistic limit data, 38 caliber, $158^{5} \mathrm{grain}$, lead round nose bullet

$\left(\mathrm{V}_{50}\right.$ expressed in feet per second)

\begin{tabular}{|c|c|c|c|c|c|c|c|c|c|c|}
\hline \multicolumn{2}{|c|}{ Unused vests } & \multicolumn{3}{|c|}{ Light wear vests } & \multicolumn{3}{|c|}{ Moderate wear vests } & \multicolumn{3}{|c|}{ Heavy wear vests } \\
\hline Sample & $\mathrm{v}_{50}$ & Sample & $v_{50}$ & $\begin{array}{l}\text { Variation } \\
\text { from unused } \\
\text { vest average } \\
\text { (percent) }\end{array}$ & Sample & $\mathrm{v}_{50}$ & $\begin{array}{l}\text { Variation } \\
\text { from unused } \\
\text { vest average } \\
\text { (percent) }\end{array}$ & Sample & $\mathrm{v}_{50}$ & $\begin{array}{l}\text { Variation } \\
\text { from unused } \\
\text { vest average } \\
\text { (percent) }\end{array}$ \\
\hline $1 F$ & 1074 & $33 \mathrm{~F}$ & 1104 & +3.2 & $10 \mathrm{~F}$ & 1108 & +3.6 & $17 F$ & 1153 & +7.8 \\
\hline $1 \mathrm{~B}$ & 1075 & 33B & 1135 & +6.1 & $10 \mathrm{~B}$ & 1165 & +8.9 & $17 \mathrm{~B}$ & 1075 & +0.5 \\
\hline $23 \mathrm{~F}$ & 1036 & $12 \mathrm{~F}^{\mathrm{c}}$ & $1126^{\mathrm{c}}$ & +5.2 & $30 \mathrm{~F}^{\mathrm{c}}$ & 1120 & +4.7 & $31 \mathrm{~F}$ & 1131 & +5.7 \\
\hline $24 \mathrm{~B}$ & 1050 & $12 B^{C}$ & ${\underline{1112^{c}}}^{\mathrm{C}}$ & +3.9 & $30 \mathrm{~B}^{\mathrm{C}}$ & 1118 & +4.5 & $31 \mathrm{~B}$ & $1152^{b}$ & +7.7 \\
\hline $4 \mathrm{~F}^{\mathrm{c}}$ & 1088 & Average & 1119 & +4.6 & Average & 1128 & +5.4 & $18 \mathrm{~F}^{\mathrm{c}}$ & 1080 & +0.9 \\
\hline $4 B^{c}$ & $\underline{1095}$ & & & & & & & $18 \mathrm{~B}^{\mathrm{C}}$ & 1074 & +0.4 \\
\hline Average & 1070 & & & & & & & $13 \mathrm{~F}^{\mathrm{c}}$ & $1159^{\mathrm{b}}$ & +8.3 \\
\hline $22 F^{a, c}$ & $1161^{a}$ & & & & & & & $13 B^{C}$ & $1129^{\mathrm{b}}$ & +5.5 \\
\hline $22 B^{a, c}$ & 1193 & & & & & & & Average & 1119 & +4.6 \\
\hline
\end{tabular}

${ }^{a_{S}}$ ample 22 contained eight layers of fabric, excluded from average.

$\mathrm{b}_{\text {Eight shot }} \mathrm{V}_{50}$ determination.

$c_{\text {Tested }}$ wet. 
Table 2. Deformation measurements, 38 caliber projectile

\begin{tabular}{|c|c|c|c|c|c|c|c|c|c|c|c|}
\hline \multicolumn{3}{|c|}{ Unused vests } & \multicolumn{3}{|c|}{ Light wear vests } & \multicolumn{3}{|c|}{ Moderate wear vests } & \multicolumn{3}{|c|}{ Heavy wear vests } \\
\hline Sample & $\begin{array}{l}\text { Average } \\
\text { Impact } \\
\text { Velocity } \\
(\mathrm{ft} / \mathrm{s})\end{array}$ & $\begin{array}{l}\text { Defor- } \\
\text { mation } \\
\text { (in) }\end{array}$ & Sample & $\begin{array}{l}\text { Average } \\
\text { Impact } \\
\text { Velocity } \\
(\mathrm{ft} / \mathrm{s})\end{array}$ & $\begin{array}{l}\text { Defor- } \\
\text { mation } \\
\text { (in) }\end{array}$ & Sample & $\begin{array}{c}\text { Average } \\
\text { Impact } \\
\text { Velocity } \\
(\mathrm{ft} / \mathrm{s})\end{array}$ & $\begin{array}{l}\text { Defor- } \\
\text { mation } \\
\text { (in) }\end{array}$ & Sample & $\begin{array}{c}\text { Average } \\
\text { Impact } \\
\text { Velocity } \\
(\mathrm{ft} / \mathrm{s})\end{array}$ & $\begin{array}{l}\text { Defor- } \\
\text { mation } \\
\text { (in) }\end{array}$ \\
\hline $1 F$ & $\begin{array}{l}895 \\
887\end{array}$ & $\begin{array}{l}1.65 \\
1.50\end{array}$ & $33 \mathrm{~F}$ & $\begin{array}{l}880 \\
893\end{array}$ & $\begin{array}{l}1.40 \\
1.40\end{array}$ & $10 \mathrm{~F}$ & $\begin{array}{l}855 \\
885\end{array}$ & $\begin{array}{l}1.50 \\
1.60\end{array}$ & $17 F$ & $\begin{array}{l}860 \\
872\end{array}$ & $\begin{array}{l}1.50 \\
1.60\end{array}$ \\
\hline $1 B$ & $\begin{array}{l}850 \\
877\end{array}$ & $\begin{array}{l}1.60 \\
1.45\end{array}$ & $33 B$ & $\begin{array}{l}874 \\
888\end{array}$ & $\begin{array}{l}1.50 \\
1.35\end{array}$ & $10 \mathrm{~B}$ & $\begin{array}{l}885 \\
898\end{array}$ & $\begin{array}{l}1.60 \\
1.45\end{array}$ & $17 \mathrm{~B}$ & $\begin{array}{l}867 \\
889\end{array}$ & $\begin{array}{l}1.50 \\
1.30\end{array}$ \\
\hline $23 \mathrm{~F}$ & $\begin{array}{l}867 \\
884\end{array}$ & $\begin{array}{l}1.50 \\
1.50\end{array}$ & $12 \mathrm{~F}^{\mathrm{a}}$ & $\begin{array}{l}892 \\
897\end{array}$ & $\begin{array}{l}1.45 \\
1.50\end{array}$ & $30 F^{a}$ & $\begin{array}{l}837 \\
839\end{array}$ & $\begin{array}{l}1.45 \\
1.65\end{array}$ & $31 \mathrm{~F}$ & $\begin{array}{l}875 \\
881\end{array}$ & $\begin{array}{l}1.45 \\
1.50\end{array}$ \\
\hline $24 B$ & $\begin{array}{l}895 \\
871\end{array}$ & $\begin{array}{l}1.50 \\
1.60\end{array}$ & $12 \mathrm{~B}^{\mathrm{a}}$ & $\begin{array}{l}842 \\
823\end{array}$ & $\begin{array}{l}1.70 \\
1.65\end{array}$ & $30 B^{a}$ & $\begin{array}{l}854 \\
837\end{array}$ & $\begin{array}{l}1.40 \\
1.60\end{array}$ & $31 B$ & $\begin{array}{l}877 \\
885\end{array}$ & $\begin{array}{l}1.35 \\
1.50\end{array}$ \\
\hline $4 \mathrm{~F}^{\mathrm{a}}$ & $\begin{array}{l}888 \\
866\end{array}$ & $\begin{array}{l}1.60 \\
1.60\end{array}$ & & & & & & & $18 F^{a}$ & $\begin{array}{l}872 \\
877\end{array}$ & $\begin{array}{l}1.60 \\
1.60\end{array}$ \\
\hline $4 \mathrm{~B}^{\mathrm{a}}$ & $\begin{array}{l}867 \\
869\end{array}$ & $\begin{array}{l}1.60 \\
1.60\end{array}$ & & & & & & & $18 \mathrm{~B}^{\mathrm{a}}$ & $\begin{array}{l}882 \\
882\end{array}$ & $\begin{array}{l}1.65 \\
1.50\end{array}$ \\
\hline $22 \mathrm{~F}^{\mathrm{a}}$ & $\begin{array}{l}896 \\
875\end{array}$ & $\begin{array}{l}1.60 \\
1.40\end{array}$ & & & & & & & $13 \mathrm{~F}^{\mathrm{a}}$ & $\begin{array}{l}821 \\
828\end{array}$ & $\begin{array}{l}1.50 \\
1.60\end{array}$ \\
\hline $22 B^{a}$ & $\begin{array}{l}881 \\
902\end{array}$ & $\begin{array}{l}1.30 \\
1.45\end{array}$ & & & & & & & $13 \mathrm{~B}^{\mathrm{a}}$ & $\begin{array}{l}837 \\
834\end{array}$ & $\begin{array}{l}1.50 \\
1.55\end{array}$ \\
\hline
\end{tabular}

$a_{\text {Tested }}$ wet.

Note: All velocity measurements rounded to nearest foot per second.

Table 3 summarizes the $V_{50}$ ballistic limit for each vest tested using 22 caliber bullets, and table 4 presents the blunt trauma deformation measurements obtained with 22 caliber bullets. 
Table 3. $V_{50}$ Ballistic Limit Data

.22 caliber, 40 grain, lead round nose bullet

$\left(V_{50}\right.$ expressed in feet per second)

\begin{tabular}{|c|c|c|c|c|c|c|c|c|c|c|}
\hline \multicolumn{2}{|c|}{ Unused vests } & \multicolumn{3}{|c|}{ Light wear vest } & \multicolumn{3}{|c|}{ Moderate wear vests } & \multicolumn{3}{|c|}{ Heavy wear vests } \\
\hline Sample & $v_{50}$ & Sample & $v_{50}$ & $\begin{array}{l}\text { Variation } \\
\text { from unused } \\
\text { vest average } \\
\text { (percent) }\end{array}$ & Sample & $\mathrm{v}_{50}$ & $\begin{array}{l}\text { Variation } \\
\text { from unused } \\
\text { vest average } \\
\text { (percent) }\end{array}$ & Sample & $\mathrm{v}_{50}$ & $\begin{array}{l}\text { Variation } \\
\text { from unused } \\
\text { vest average } \\
\text { (percent) }\end{array}$ \\
\hline $2 \mathrm{~F}$ & 1179 & $15 F$ & 1219 & +1.6 & $32 \mathrm{~F}$ & 1238 & +3.2 & $20 \mathrm{~F}$ & 1160 & -3.3 \\
\hline $2 \mathrm{~B}$ & 1203 & $15 B$ & 1267 & +5.6 & $32 \mathrm{~B}$ & 1224 & +2.0 & $20 \mathrm{~B}$ & 1172 & -2.3 \\
\hline $25 \mathrm{~F}$ & 1200 & $21 F^{b}$ & 1214 & +1.2 & $7 F^{b}$ & 1183 & -1.4 & $8 \mathrm{~F}$ & 1200 & 0 \\
\hline $25 B$ & 1217 & $21 B^{b}$ & $\underline{1239}$ & +3.3 & $7 \mathrm{~B}^{\mathrm{b}}$ & $\underline{1251}$ & $\underline{+4.3}$ & $8 \mathrm{~B}$ & 1205 & +0.4 \\
\hline $3 F^{b}$ & 1169 & Average & 1235 & +2.9 & Average & 1224 & +2.0 & $9 \mathrm{~F}$ & 1183 & -1.4 \\
\hline $3 \mathrm{~B}^{\mathrm{b}}$ & 1164 & & & & & & & $9 \mathrm{~B}$ & 1154 & -3.8 \\
\hline $26 \mathrm{~B}^{\mathrm{b}}$ & 1260 & & & & & & & $6 \mathrm{~F}$ & 1182 & -1.5 \\
\hline $27 \mathrm{~F}^{\mathrm{b}}$ & $\underline{1210}$ & & & & & & & $6 \mathrm{~B}$ & $1137^{a}$ & $\underline{-5.3}$ \\
\hline Average & 1200 & & & & & & & Average & 1174 & -2.2 \\
\hline \multicolumn{2}{|c|}{$\begin{array}{l}\text { Dry retest, } \\
\text { not included } \\
\text { in average }\end{array}$} & & & & & & & \multicolumn{3}{|c|}{$\begin{array}{l}\text { Initial wet test, } \\
\text { not included } \\
\text { in average }\end{array}$} \\
\hline $3 F$ & 1102 & & & & & & & $9 F^{b}$ & 1183 & -1.4 \\
\hline \multirow[t]{3}{*}{ 3в } & 1182 & & & & & & & $9 B^{b}$ & 1099 & -8.4 \\
\hline & & & & & & & & $6 \mathrm{~F}^{\mathrm{b}}$ & $1075^{a}$ & -10.4 \\
\hline & & & & & & & & $6 \mathrm{~B}^{\mathrm{b}}$ & $1145^{a}$ & -4.6 \\
\hline
\end{tabular}

${ }^{a}$ Eight shot $\mathrm{v}_{50}$ determination.

$b_{\text {Tested wet. }}$ 
Table 4. Deformation measurements, 22 caliber projectile

\begin{tabular}{|c|c|c|c|c|c|c|c|c|c|c|c|}
\hline \multicolumn{3}{|c|}{ Unused vests } & \multicolumn{3}{|c|}{ Light wear vests } & \multicolumn{3}{|c|}{ Moderate wear vests } & \multicolumn{3}{|c|}{ Heavy wear vests } \\
\hline Sample & $\begin{array}{c}\text { Average } \\
\text { Impact } \\
\text { Velocity } \\
(\mathrm{ft} / \mathrm{s})\end{array}$ & $\begin{array}{l}\text { Defor- } \\
\text { mation } \\
\text { (in) }\end{array}$ & Sample & $\begin{array}{l}\text { Average } \\
\text { Impact } \\
\text { Velocity } \\
\text { (ft/s) }\end{array}$ & $\begin{array}{l}\text { Defor- } \\
\text { mation } \\
\text { (in) }\end{array}$ & Sample & $\begin{array}{c}\text { Average } \\
\text { Impact } \\
\text { Velocity } \\
\text { (ft/s) }\end{array}$ & $\begin{array}{l}\text { Defor- } \\
\text { mation } \\
\text { (in) }\end{array}$ & Sample & $\begin{array}{l}\text { Average } \\
\text { Impact } \\
\text { Velocity } \\
(\mathrm{ft} / \mathrm{s})\end{array}$ & $\begin{array}{l}\text { Defor- } \\
\text { mation } \\
\text { (in) }\end{array}$ \\
\hline $2 F$ & $\begin{array}{l}1064 \\
1063\end{array}$ & $\begin{array}{l}0.80 \\
0.85\end{array}$ & $15 \mathrm{~F}$ & $\begin{array}{l}1054 \\
1115\end{array}$ & $\begin{array}{l}0.80 \\
0.90\end{array}$ & $32 \mathrm{~F}$ & $\begin{array}{l}1029 \\
1085\end{array}$ & $\begin{array}{l}0.90 \\
0.85\end{array}$ & $20 \mathrm{~F}$ & $\begin{array}{l}1075 \\
1067\end{array}$ & $\begin{array}{l}0.70 \\
0.65\end{array}$ \\
\hline 2B & $\begin{array}{l}1096 \\
1063\end{array}$ & $\begin{array}{l}0.90 \\
0.85\end{array}$ & $15 B$ & $\begin{array}{l}1101 \\
1083\end{array}$ & $\begin{array}{l}0.55 \\
0.60\end{array}$ & $32 \mathrm{~B}$ & $\begin{array}{l}1066 \\
1068\end{array}$ & $\begin{array}{l}0.80 \\
0.80\end{array}$ & $20 \mathrm{~B}$ & $\begin{array}{l}1057 \\
1063\end{array}$ & $\begin{array}{l}0.80 \\
0.70\end{array}$ \\
\hline $25 \mathrm{~F}$ & $\begin{array}{l}1077 \\
1054\end{array}$ & $\begin{array}{l}0.85 \\
0.80\end{array}$ & $21 F^{a}$ & $\begin{array}{l}1058 \\
1083\end{array}$ & $\begin{array}{l}0.80 \\
0.85\end{array}$ & $7 F^{a}$ & $\begin{array}{l}1054 \\
1085\end{array}$ & $\begin{array}{l}0.80 \\
0.80\end{array}$ & $8 F$ & $\begin{array}{l}1058 \\
1081\end{array}$ & $\begin{array}{l}0.85 \\
0.75\end{array}$ \\
\hline $25 \mathrm{~B}$ & $\begin{array}{l}1051 \\
1070\end{array}$ & $\begin{array}{l}0.80 \\
0.85\end{array}$ & $21 \mathrm{~B}^{\mathrm{a}}$ & $\begin{array}{l}1066 \\
1066\end{array}$ & $\begin{array}{l}0.80 \\
0.75\end{array}$ & $7 \mathrm{~B}^{\mathrm{a}}$ & $\begin{array}{l}1040 \\
1038\end{array}$ & $\begin{array}{l}0.80 \\
0.85\end{array}$ & 8B & $\begin{array}{l}1040 \\
1081\end{array}$ & $\begin{array}{l}0.75 \\
0.25\end{array}$ \\
\hline $3 F^{2}$ & $\begin{array}{l}1049 \\
1071\end{array}$ & $\begin{array}{l}0.85 \\
0.95\end{array}$ & & & & & & & $9 F^{a}$ & $\begin{array}{l}1059 \\
1053\end{array}$ & $\begin{array}{l}0.20 \\
0.75\end{array}$ \\
\hline $3 \mathrm{~B}^{\mathrm{a}}$ & $\begin{array}{l}1071 \\
1103\end{array}$ & $\begin{array}{l}0.75 \\
0.80\end{array}$ & & & & & & & $9 B^{a}$ & $\begin{array}{l}1095 \\
1060\end{array}$ & $\begin{array}{l}0.80 \\
0.80\end{array}$ \\
\hline $26 \mathrm{~B}^{\mathrm{a}}$ & $\begin{array}{l}1083 \\
1073\end{array}$ & $\begin{array}{l}0.75 \\
0.70\end{array}$ & & & & & & & $6 \mathrm{~F}^{\mathrm{a}}$ & $\begin{array}{l}1054 \\
1049\end{array}$ & $\begin{array}{l}\mathrm{b} \\
\mathrm{b}\end{array}$ \\
\hline $27 \mathrm{~F}^{\mathrm{a}}$ & $\begin{array}{l}1101 \\
1038 \\
1064\end{array}$ & $\begin{array}{l}0.85 \\
0.75 \\
0.75\end{array}$ & & & & & & & $6 \mathrm{~B}^{\mathrm{a}}$ & $\begin{array}{l}1068 \\
1085\end{array}$ & $\begin{array}{l}0.80 \\
0.65\end{array}$ \\
\hline
\end{tabular}

\footnotetext{
$a_{\text {Tested }}$ wet.

${ }^{b}$ No measurement, test round penetrated armor.

Note: All velocity measurements rounded to nearest foot per second.
}

Table 5 expands upon the ballistic limit data summarized in table 1, to include the velocity of the highest velocity nonpenetrating round $\left(H_{p}\right)$, the velocity of the lowest velocity complete penetration round $\left(L_{C}\right)$ and the velocity range of mixed results of penetrating and nonpenetrating rounds for 38 caliber bullets. 
Table 5. Complete ballistic limit data, 38 caliber

\begin{tabular}{|c|c|c|c|c|c|c|c|c|c|}
\hline \multicolumn{5}{|c|}{ Unused vests } & \multicolumn{5}{|c|}{ Medium wear vests } \\
\hline $\begin{array}{c}\text { Sample } \\
\text { no. }\end{array}$ & $\begin{array}{r}V_{50} \\
(\mathrm{ft} / \mathrm{s}) \\
\end{array}$ & $\begin{array}{c}{ }^{H_{p}} \\
(\mathrm{ft} / \mathrm{s}) \\
\end{array}$ & $\begin{array}{c}I_{C} \\
(f t / s) \\
\end{array}$ & $\begin{array}{l}\text { Range } \\
\text { mixed } \\
(\mathrm{ft} / \mathrm{s}) \\
\end{array}$ & $\begin{array}{c}\text { Sample } \\
\text { no. }\end{array}$ & $\begin{array}{l}v_{50} \\
(\mathrm{ft} / \mathrm{s}) \\
\end{array}$ & $\begin{array}{c}{ }^{H} p \\
(f t / s) \\
\end{array}$ & $\begin{array}{c}I_{C} \\
(\mathrm{ft} / \mathrm{s})\end{array}$ & $\begin{array}{l}\text { Range } \\
\text { mixed } \\
(\mathrm{ft} / \mathrm{s}) \\
\end{array}$ \\
\hline $\begin{array}{r}1 \mathrm{~F} \\
1 \mathrm{~B} \\
23 \mathrm{~F} \\
24 \mathrm{~B} \\
4 \mathrm{~F}^{\mathrm{a}} \\
4 \mathrm{~B}^{\mathrm{a}}\end{array}$ & $\begin{array}{l}1074 \\
1075 \\
1036 \\
1050 \\
1088 \\
1095\end{array}$ & $\begin{array}{l}1113 \\
1073 \\
1083 \\
1079 \\
1109 \\
1087\end{array}$ & $\begin{array}{r}1031 \\
1075 \\
988 \\
1036 \\
1064 \\
1105\end{array}$ & $\begin{array}{r}82 \\
N / A \\
95 \\
43 \\
45 \\
N / A\end{array}$ & $\begin{array}{r}10 F \\
10 B \\
30 F^{a} \\
30 B^{a}\end{array}$ & $\begin{array}{l}1108 \\
1165 \\
1120 \\
1118\end{array}$ & $\begin{array}{l}1163 \\
1220 \\
1165 \\
1138\end{array}$ & $\begin{array}{l}1091 \\
1101 \\
1093 \\
1093\end{array}$ & $\begin{array}{r}72 \\
119 \\
72 \\
45\end{array}$ \\
\hline $\begin{array}{l}22 A^{a} \\
22 B^{a}\end{array}$ & $\begin{array}{l}1161 \\
1193\end{array}$ & $\begin{array}{l}1147 \\
1238\end{array}$ & $\begin{array}{l}1170 \\
1149\end{array}$ & $\begin{array}{r}\text { N/A } \\
89\end{array}$ & & & & & \\
\hline
\end{tabular}

\begin{tabular}{ccccr}
\hline \multicolumn{5}{c}{ Light wear vests } \\
\hline $\begin{array}{c}\text { Sample } \\
\text { no. }\end{array}$ & $\begin{array}{c}\mathrm{V}_{50} \\
(\mathrm{ft} / \mathrm{s})\end{array}$ & $\begin{array}{c}\mathrm{H}_{\mathrm{p}} \\
(\mathrm{ft} / \mathrm{s})\end{array}$ & $\begin{array}{c}\mathrm{L}_{\mathrm{C}} \\
(\mathrm{ft} / \mathrm{s})\end{array}$ & $\begin{array}{r}\text { Range } \\
\text { mixed } \\
(\mathrm{ft} / \mathrm{s})\end{array}$ \\
\hline & & & & \\
$33 \mathrm{~F}$ & 1104 & 1181 & 1062 & 119 \\
$33 \mathrm{~B}$ & 1135 & 1167 & 1111 & 56 \\
$12 \mathrm{~F}^{\mathrm{a}}$ & 1126 & 1113 & 1113 & 0 \\
$12 \mathrm{~B}$ & 1112 & 1097 & 1095 & 2 \\
& & & & \\
\end{tabular}

\begin{tabular}{ccccr}
\hline \multicolumn{5}{c}{ Heavy wear vests } \\
$\begin{array}{c}\text { Sample } \\
\text { no. }\end{array}$ & $\begin{array}{c}\mathrm{V}_{50} \\
(\mathrm{ft} / \mathrm{s})\end{array}$ & $\begin{array}{c}\mathrm{H}_{\mathrm{p}} \\
(\mathrm{ft} / \mathrm{s})\end{array}$ & $\begin{array}{c}\mathrm{L}_{\mathrm{C}} \\
(\mathrm{ft} / \mathrm{s})\end{array}$ & $\begin{array}{r}\text { Range } \\
\mathrm{mixed} \\
(\mathrm{ft} / \mathrm{s})\end{array}$ \\
\hline & & & & \\
$17 \mathrm{~F}$ & 1153 & 1163 & 1154 & 9 \\
$17 \mathrm{~B}$ & 1075 & 1107 & 1062 & 45 \\
$31 \mathrm{~F}$ & $1131 \mathrm{~b}$ & 1174 & 1060 & 114 \\
$31 \mathrm{~B}$ & $1152^{\mathrm{b}}$ & 1208 & 1132 & 76 \\
$18 \mathrm{~F}$ & 1080 & 1097 & 1064 & 33 \\
$18 \mathrm{~B} a$ & $1074 \mathrm{~b}$ & 1097 & 1062 & 35 \\
$13 \mathrm{Fa}$ & $1159 \mathrm{~b}$ & 1222 & 1158 & 64 \\
$13 \mathrm{~B}$ & $1129 \mathrm{~b}$ & 1210 & 1103 & 107 \\
\hline
\end{tabular}

$\mathrm{a}_{\text {Tested }}$ wet.

$b_{\text {Eight shot }} \mathrm{v}_{50}$.

Table 6 expands upon the ballistic limit data summarized in table 3 , including $\mathrm{H}_{p}$. $L_{C^{\prime}}$ and range of mixed velocities for 22 caliber bullets. 
Table 6. Complete ballistic limit data, 22 caliber

\begin{tabular}{|c|c|c|c|c|}
\hline \multicolumn{5}{|c|}{ Unused vests } \\
\hline $\begin{array}{c}\text { Sample } \\
\text { no. }\end{array}$ & $\begin{array}{l}V_{50} \\
(\mathrm{ft} / \mathrm{s}) \\
\end{array}$ & $\begin{array}{c}{ }^{H} p \\
( \pm t / s)\end{array}$ & $\begin{array}{c}L_{c} \\
( \pm t / s)\end{array}$ & $\begin{array}{l}\text { Range } \\
\text { mixed } \\
(\mathrm{ft} / \mathrm{s}) \\
\end{array}$ \\
\hline $\begin{array}{r}2 \mathrm{~F} \\
2 \mathrm{~B} \\
25 \mathrm{~F} \\
25 \mathrm{~B} \\
3 \mathrm{~F}^{\mathrm{a}} \\
3 \mathrm{~B}^{\mathrm{a}} \\
26 \mathrm{~B}^{\mathrm{a}} \\
27 \mathrm{~F}^{\mathrm{a}}\end{array}$ & $\begin{array}{l}1179 \\
1203 \\
1200 \\
1217 \\
1169 \\
1164 \\
1260 \\
1210\end{array}$ & $\begin{array}{l}1202 \\
1238 \\
1245 \\
1255 \\
1176 \\
1195 \\
1288 \\
1238\end{array}$ & $\begin{array}{l}1165 \\
1188 \\
1174 \\
1176 \\
1152 \\
1143 \\
1232 \\
1160\end{array}$ & $\begin{array}{l}37 \\
50 \\
71 \\
79 \\
24 \\
52 \\
56 \\
78\end{array}$ \\
\hline \multicolumn{5}{|c|}{ Retest (Dry) } \\
\hline $\begin{array}{l}3 F \\
3 B\end{array}$ & $\begin{array}{l}1102 \\
1182\end{array}$ & $\begin{array}{l}1085 \\
1220\end{array}$ & $\begin{array}{l}1085 \\
1170\end{array}$ & $\begin{array}{r}0 \\
50\end{array}$ \\
\hline
\end{tabular}

\begin{tabular}{lcccc}
\hline \multicolumn{5}{c}{ Medium wear vests } \\
\hline $\begin{array}{c}\text { Sample } \\
\text { no. }\end{array}$ & $\begin{array}{c}\mathrm{V}_{50} \\
(\mathrm{ft} / \mathrm{s})\end{array}$ & $\begin{array}{c}\mathrm{H}_{\mathrm{p}} \\
(\mathrm{ft} / \mathrm{s})\end{array}$ & $\begin{array}{c}\mathrm{L}_{\mathrm{C}} \\
(\mathrm{ft} / \mathrm{s})\end{array}$ & $\begin{array}{c}\text { Range } \\
\text { mixed } \\
(\mathrm{ft} / \mathrm{s})\end{array}$ \\
\hline & & & & \\
$32 \mathrm{~F}$ & 1238 & 1268 & 1215 & 53 \\
$32 \mathrm{~B}$ & 1224 & 1258 & 1192 & 66 \\
$7 \mathrm{~F}^{\mathrm{a}}$ & 1183 & 1156 & 1154 & 02 \\
$7 \mathrm{~B}^{\mathrm{a}}$ & 1251 & 1261 & 1230 & 31 \\
& & & & \\
& & & & \\
& & & & \\
\end{tabular}

\begin{tabular}{lcccc}
\hline \multicolumn{5}{c}{ Light wear vests } \\
\hline $\begin{array}{c}\text { Sample } \\
\text { no. }\end{array}$ & $\begin{array}{c}\mathrm{V}_{50} \\
(\mathrm{Et} / \mathrm{s})\end{array}$ & $\begin{array}{c}\mathrm{H}_{\mathrm{p}} \\
(\mathrm{ft} / \mathrm{s})\end{array}$ & $\begin{array}{c}\mathrm{L}_{\mathrm{C}} \\
(\mathrm{ft} / \mathrm{s})\end{array}$ & $\begin{array}{c}\text { Range } \\
\text { mixed } \\
(\mathrm{ft} / \mathrm{s})\end{array}$ \\
\hline & & & & \\
$15 \mathrm{~F}$ & 1219 & 1220 & 1220 & 0 \\
$15 \mathrm{~B}$ & 1267 & 1290 & 1260 & 30 \\
$21 \mathrm{~F}^{\mathrm{a}}$ & 1214 & 1242 & 1198 & 44 \\
$21 \mathrm{~B}^{\mathrm{a}}$ & 1239 & 1248 & 1240 & 8
\end{tabular}

\begin{tabular}{|c|c|c|c|c|}
\hline \multicolumn{5}{|c|}{ Heavy wear vests } \\
\hline $\begin{array}{c}\text { Sample } \\
\text { no. }\end{array}$ & $\begin{array}{c}V_{50} \\
( \pm t / s)\end{array}$ & $\begin{array}{c}{ }_{\mathrm{H}} \\
(\mathrm{ft} / \mathrm{s}) \\
\end{array}$ & $\begin{array}{c}L_{C} \\
( \pm t / s)\end{array}$ & $\begin{array}{l}\text { Range } \\
\text { mixed } \\
(\mathrm{ft} / \mathrm{s})\end{array}$ \\
\hline $\begin{array}{l}20 \mathrm{~F} \\
20 \mathrm{~B} \\
8 \mathrm{~F} \\
8 \mathrm{~B} \\
9 \mathrm{~F}^{\mathrm{a}} \\
9 \mathrm{~B}^{\mathrm{a}} \\
6 \mathrm{~F}^{\mathrm{a}} \\
6 \mathrm{~B}\end{array}$ & $\begin{array}{l}1160 \\
1172 \\
1200 \\
1205 \\
1183 \\
1099 \mathrm{~b} \\
1075^{\mathrm{b}} \\
1145^{\mathrm{b}}\end{array}$ & $\begin{array}{l}1147 \\
1174 \\
1222 \\
1220 \\
1181 \\
1147 \\
1091 \\
1172\end{array}$ & $\begin{array}{l}1145 \\
1172 \\
1176 \\
1195 \\
1154 \\
1075 \\
1049 \\
1115\end{array}$ & $\begin{array}{r}2 \\
2 \\
46 \\
25 \\
27 \\
72 \\
42 \\
57\end{array}$ \\
\hline \multicolumn{5}{|c|}{ Retest (Dry) } \\
\hline $\begin{array}{l}9 F \\
9 B \\
6 F \\
6 B\end{array}$ & $\begin{array}{l}1183 \\
1154 \\
1182 \mathrm{~b} \\
1137^{\circ}\end{array}$ & $\begin{array}{l}1198 \\
1178 \\
1192 \\
1165\end{array}$ & $\begin{array}{l}1160 \\
1136 \\
1155 \\
1143\end{array}$ & $\begin{array}{l}38 \\
42 \\
37 \\
22\end{array}$ \\
\hline
\end{tabular}

a Tested wet.

$\mathrm{b}_{\text {Eight shot }} \mathrm{v}_{50^{\circ}}$

Appendix A presents the raw data for each sample that was tested using 38 caliber projectiles, noting the impact velocity of each bullet that was fired to determine the $\mathrm{v}_{50}$ ballistic limit, whether or not it penetrated, and identifies those test rounds used to calculate the $\mathrm{V}_{50}$ ballistic limit. Test rounds associated with deformation testing (see tables 2 and 4 ) are not included. Appendix B presents the same penetration data as appendix A but for 22 caliber projectiles under the same limitations as appendix A. 
Those test rounds reported in appendix $A$ and $B$ that were not used to obtain the $V_{50}$ ballistic limit were excluded from calculation for a variety of reasons, including 1) hitting too close to an edge or prior hit, 2) projectile yaw, and 3 ) outside of the desired maximum velocity range.

\section{DISCUSSION}

When tested following the procedures of NILECJ-STD-0101.01, the deformation measurements that were made for all samples were well within the specified maximum limit of 1.73 in. There was no apparent difference between dry testing and wet testing. From these tests we concluded that impact deformation does not appear to hold promise as an early indicator of loss of ballistic resistant efficiency.

The interpretation of the $V_{50}$ ballistic limit data for the vests that were evaluated can be considered somewhat subjective because the data are too limited to permit rigorous statistical analysis. However, the following was derived from this series of tests.

The average $V_{50}$ ballistic limit for the 38 caliber test rounds of the 10 year old unused armor is $1070 \mathrm{ft} / \mathrm{s}$, excluding vest 22, which consists of eight layers of Kevlar. The standard deviation of the $v_{50}$ is $22.6 \mathrm{ft} / \mathrm{s}$, with an overall range of $59 \mathrm{ft} / \mathrm{s}$. There is no obvious difference within any of the four wear groups (new, light wear, moderate wear, or heavy wear) between tests conducted with the armor wet or dry.

When the $\mathrm{V}_{50}$ ballistic limit of each of the used vests is compared to the average of the unused vests of the same construction, in all cases the used vest has a higher $\mathrm{V}_{50}$

When the average $v_{50}$ of the three used vest groups (wet and dry testing averaged together) is compared with that of the unused armor, each of the three used vest groups exhibit $v_{50}$ roughly 5 percent higher. 
$\mathrm{V}_{50}$ ballistic limit data for the 158 -grain lead round nose bullet was not reported in testing conducted by Edgewood Arsenal during the earlier LEAA development effort; however, partial data [4] imply a $v_{50}$ ballistic limit on the order of $1000 \mathrm{ft} / \mathrm{s}$. Since the tests were conducted with fabric not sewn together, one would expect a higher $v_{50}$ for the finished vests that were tested, and the values of $v_{50}$ ballistic limit that were obtained are consistent with the earlier data.

It is perhaps of more interest to examine the test results in terms of the velocity of the lowest complete penetration for each panel, for this gives a better idea of absolute ballistic resistance relative to the rated threat level. The test velocity for the determination of penetration for a Type $I$ vest is $850 \pm 50 \mathrm{ft} / \mathrm{s}$, or a maximum velocity of $900 \mathrm{ft} / \mathrm{s}$. With one exception, all of the vests that were tested demonstrated a velocity for the lowest velocity complete penetration more than $150 \mathrm{ft} / \mathrm{s}$ above that required for minimum performance. Even the poorest performing armor sample (vest 23 front) exceeded the $900 \mathrm{ft} / \mathrm{s}$ requirement by a velocity of $88 \mathrm{ft} / \mathrm{s}$.

As with the 38 caliber testing of $v_{50}$ ballistic limit, there did not appear to be any overall difference between 22 caliber tests conducted wet and dry, other than discussed below. The overall average $v_{50}$ ballistic limit using the 22 caliber 40 grain lead round nose bullet was $1200 \mathrm{ft} / \mathrm{s}$ with a standard deviation of $31 \mathrm{ft} / \mathrm{s}$ and an overall range of $96 \mathrm{ft} / \mathrm{s}$. Earlier data from Edgewood Arsenal [3] reports a $v_{50}$ ballistic limit for seven layers of Kevlar 1000 denier fabric of $1084 \mathrm{ft} / \mathrm{s}$. Again, these tests used fabric only and the ballistic limit of the fabricated vests would be expected to exceed that reported. The same Edgewood report presents a $V_{50}$ ballistic limit of seven layers of 1140 denier Kevlar of $1213 \mathrm{ft} / \mathrm{s}$. This fabric is similar to 1000 denier in ballistic efficiency, although, it was not treated for water repellency. Since the overall ballistic efficiency of treated Kevlar is known to be less than untreated, the average $V_{50}$ of $1200 \mathrm{ft} / \mathrm{s}$ is reasonable for the unused armor that was tested in this program.

When first tested wet, the front panel of vest 6 was penetrated at velocities of 1054 and $1049 \mathrm{ft} / \mathrm{s}$ and the $V_{50}$ ballistic limit was $1075 \mathrm{ft} / \mathrm{s}$; well below the $1100 \mathrm{ft} / \mathrm{s}$ upper limit of threat Type I protection $(1050 \pm 50 \mathrm{ft} / \mathrm{s})$. Likewise, the $V_{50}$ ballistic limit of the back panel of vest 9 was $1099 \mathrm{ft} / \mathrm{s}$ which is marginal performance at best. Since all other vests tested with 22 caliber bullets were found to have $v_{50}$ ballistic 
limits well in excess of $1100 \mathrm{ft} / \mathrm{s}$, the data for vests 6 and 9 were suspect. Similarly, vest 3 , also tested wet, appeared to have a somewhat low $v_{50}$ ballistic limit, when compared to the other unused vests.

Vests 3,6 , and 9 were allowed to dry in a well ventilated, controlled environment for several days, and once feeling dry to touch, were retested dry for ballistic limit using the 22 caliber ammunition. The two panels in most question, $9 B$ and $6 \mathrm{~F}$, when retested $d r y$, demonstrated $v_{50}$ ballistic limits well above $1100 \mathrm{ft} / \mathrm{s}$, as did the lowest velocity complete penetrating rounds. The other parts of these vests continued to demonstrate satisfactory $V_{50}$ ballistic limit and lowest complete penetrating velocity, when dry.

The retest of vest 3 was not conclusive. The $v_{50}$ ballistic limit of the back panel increased slightly when retested; however, that of the front panel decreased. While the placement of the bullet impacts during retest were sufficiently distant from prior impact locations to constitute fair hits, it remains possible that the previous testing prevented obtaining valid $V_{50}$ data in the second set of tests (see fig. 2 ).

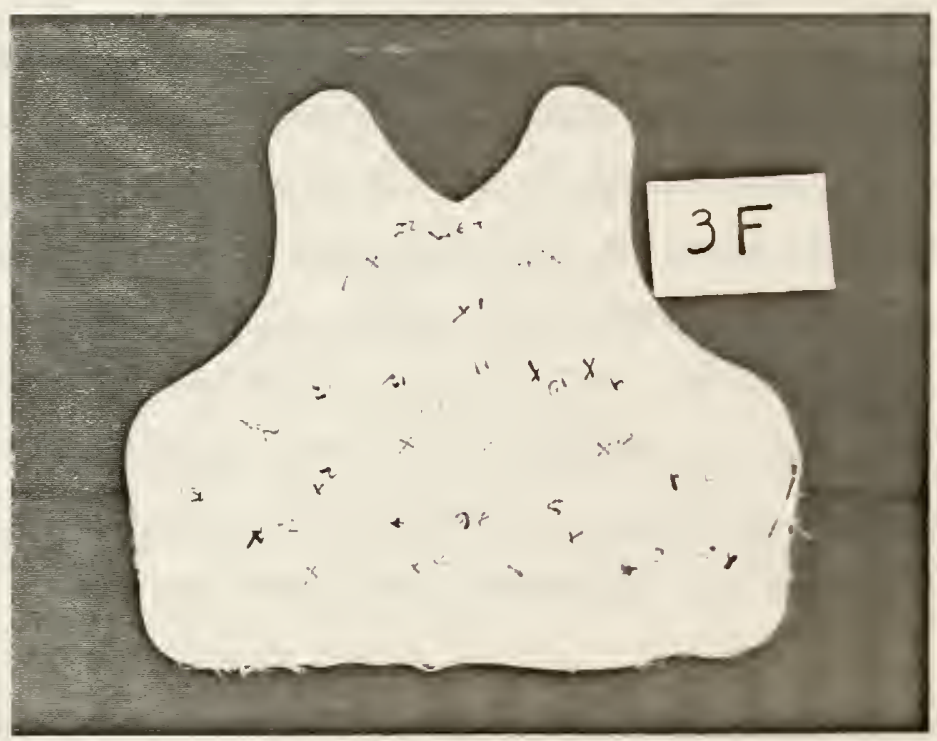

Figure 2. Front of vest 3 after being shot. 
Overall, as with the 38 caliber testing, the $v_{50}$ ballistic limit of the light and moderate wear samples tested with 22 caliber bullets increased when compared with the average of the unused samples. The heavily worn vests had an average $v_{50}$ ballistic limit of $1174 \mathrm{ft} / \mathrm{s}$ (using data for vest 6 and 9 tested dry), 2.2 percent less than the average $1200 \mathrm{ft} / \mathrm{s}$ ballistic limit of the unused vests.

\section{CONCLUSIONS}

Body armor manufactured from Kevlar fabric retains full ballistic efficiency when stored under typical warehouse conditions for periods of time in excess of 10 years. It may well be, as anticipated, that such armor has an unlimited shelf life.

Deformation measurements using wet or dry armor do not appear to provide significant information as a means of identifying decreased ballistic efficiency of used armor manufactured from Kevlar fabric.

Two of the sample vests that were tested for ballistic limit did not appear to have adequate waterproofing. It is not known whether this was a consequence of heavy wear or improper water repellent treatment at the time of manufacture.

Both the 38 caliber and 22 caliber ballistic limit data support an apparent trend of improved ballistic efficiency as a consequence of light to moderate wear and possibly a slight decrease in ballistic efficiency as a result of heavy wear. The limited data that were obtained and the variation of ballistic efficiency within lots of Kevlar fabric is such that it is very difficult to say with certainty that the difference between the $v_{50}$ of unused and heavily used vests is solely a consequence of wear, or due to the individual samples. Recent data obtained from the U.S. Army [5] for $V_{50}$ ballistic limit of 16 production lots of Kevlar fabric (12-layer test samples tested using the 22 caliber fragment simulator) demonstrate ranges of $v_{50}$ ballistic limits of more than 6 percent between individual lots.

The possible trend of decreased ballistic efficiency of armor following heavy use, coupled with the identification of at least two armor panels that lacked waterproofing, 
strongly suggest that it is prudent for any police department to inspect the vests worn by its officers on at least an annual basis. Representative samples of vests showing extremely heavy wear should be tested for ballistic performance. Such tests should be conducted with the vests in the wet condition. In the interest of minimizing testing cost, it is recommended that such testing be limited to the six shot test sequence specified by the current edition of the NIJ Standard using a single test round (22 caliber for Type I vests and $9 \mathrm{~mm}$ for Level IIA, II, and IIIA vests).

\section{REFERENCES}

[1] Body armor field test and evaluation final report. Vol. I, Executive Summary. Aerospace Corporation, Law Enforcement and Telecommunications Division, Washington, DC; 1977 september.

[2] Ballistic test for armor. Military Standard MIL-STD-662D. U.S. Army Materials and Mechanics Research Center, Watertown, MA 02172; 1984 March 19.

[3] Ballistic resistance of police body armor. NILECJ-STD-0101.01. National Institute of Justice, U.S. Department of Justice, Washington, DC 20531; 1978 March. Superseded by NIJ Standard-0101.02, Ballistic Resistance of Police Body Armor dated March 1985.

[4] Prather, R. N., Swain, C. L., Hawkins, C. E. Back face signatures of soft body armors and the associated trauma effects. RCSL-TR-77-55. Chemical Systems Laboratory, Aberdeen Proving Ground, MD; 1977 November.

[5] Personal communication with the Defense Personnel Support Center, Philadelphia, PA. 
APPENDIX A

$\mathrm{V}_{50}$ Ballistic Limit Test Data

Seven Layer Kevlar Soft Body Armor

38 Caliber, 158 Grain, Lead Round Nose Projectile 
Sample 1 (unused); tested dry

\begin{tabular}{|c|c|c|c|}
\hline \multicolumn{4}{|c|}{ Front } \\
\hline \multicolumn{2}{|c|}{$\begin{array}{c}\text { Partial } \\
\text { penetration }\end{array}$} & \multicolumn{2}{|c|}{$\begin{array}{l}\text { Complete } \\
\text { penetration }\end{array}$} \\
\hline $\begin{array}{l}\text { Round } \\
\text { no. }\end{array}$ & $\begin{array}{c}\text { Average } \\
\text { velocity } \\
\text { (ft/s) }\end{array}$ & $\begin{array}{l}\text { Round } \\
\text { no. }\end{array}$ & $\begin{array}{c}\text { Average } \\
\text { velocity } \\
\text { (ft/s) }\end{array}$ \\
\hline 4 & 1026 & 3 & 1143 \\
\hline 5 & 1081 & 6 & 1218 \\
\hline 8 & $1077^{a}$ & 7 & $1085 \mathrm{a}$ \\
\hline 10 & $1081 a$ & 9 & $1081^{a}$ \\
\hline 13 & $1075 a$ & 11 & $1064^{\mathrm{a}}$ \\
\hline 14 & $1113^{a}$ & 12 & $1031^{\mathrm{a}}$ \\
\hline 16 & $1036 \mathrm{a}$ & 15 & $1097^{a}$ \\
\hline
\end{tabular}

\begin{tabular}{|c|c|c|c|}
\hline \multicolumn{4}{|c|}{ Back } \\
\hline \multicolumn{2}{|c|}{$\begin{array}{c}\text { Partial } \\
\text { penetration }\end{array}$} & \multicolumn{2}{|c|}{$\begin{array}{c}\text { Complete } \\
\text { penetration }\end{array}$} \\
\hline $\begin{array}{l}\text { Round } \\
\text { no. }\end{array}$ & $\begin{array}{c}\text { Average } \\
\text { velocity } \\
(\mathrm{ft} / \mathrm{s})\end{array}$ & $\begin{array}{c}\text { Round } \\
\text { no. }\end{array}$ & $\begin{array}{c}\text { Average } \\
\text { velocity } \\
\text { (ft/s) }\end{array}$ \\
\hline 3 & 1005 & 6 & $1075^{a}$ \\
\hline 4 & 995 & 8 & $1138^{a}$ \\
\hline 5 & $1073^{a}$ & 10 & $1089^{a}$ \\
\hline 7 & 906 & 11 & $1111^{\mathrm{a}}$ \\
\hline 9 & $1022^{a}$ & 13 & 1178 \\
\hline 12 & $1058^{a}$ & 15 & 1158 \\
\hline 14 & $1026^{a}$ & 16 & $1124^{a}$ \\
\hline 17 & $1031^{\mathrm{a}}$ & & \\
\hline
\end{tabular}

Sample 4 (unused); tested wet

\begin{tabular}{|c|c|c|c|}
\hline \multicolumn{4}{|c|}{ Front } \\
\hline \multicolumn{2}{|c|}{$\begin{array}{c}\text { Partial } \\
\text { penetration }\end{array}$} & \multicolumn{2}{|c|}{$\begin{array}{l}\text { Complete } \\
\text { penetration }\end{array}$} \\
\hline $\begin{array}{l}\text { Round } \\
\text { no. }\end{array}$ & $\begin{array}{c}\text { Average } \\
\text { velocity } \\
(\mathrm{ft} / \mathrm{s})\end{array}$ & $\begin{array}{l}\text { Round } \\
\text { no. }\end{array}$ & $\begin{array}{c}\text { Average } \\
\text { velocity } \\
\text { (ft/s) }\end{array}$ \\
\hline 4 & $1109^{a}$ & 3 & 1202 \\
\hline 9 & $1073^{a}$ & 5 & 1188 \\
\hline 10 & $1068^{a}$ & 6 & $1070^{a}$ \\
\hline 11 & $1051^{\mathrm{a}}$ & 7 & $1149^{a}$ \\
\hline \multirow[t]{3}{*}{12} & $1022^{a}$ & 8 & $1130 a$ \\
\hline & & 13 & $1149^{a}$ \\
\hline & & 14 & $1064^{\mathrm{a}}$ \\
\hline
\end{tabular}

\begin{tabular}{|c|c|c|c|}
\hline \multicolumn{4}{|c|}{ Back } \\
\hline \multicolumn{2}{|c|}{$\begin{array}{c}\text { Partial } \\
\text { penetration }\end{array}$} & \multicolumn{2}{|c|}{$\begin{array}{c}\text { Complete } \\
\text { penetration }\end{array}$} \\
\hline $\begin{array}{l}\text { Round } \\
\text { no. }\end{array}$ & $\begin{array}{c}\text { Average } \\
\text { velocity } \\
(\mathrm{ft} / \mathrm{s})\end{array}$ & $\begin{array}{l}\text { Round } \\
\text { no. }\end{array}$ & $\begin{array}{c}\text { Average } \\
\text { velocity } \\
(\mathrm{ft} / \mathrm{s})\end{array}$ \\
\hline 4 & 1014 & 3 & $1143^{a}$ \\
\hline 5 & 1020 & 6 & $1156^{a}$ \\
\hline 8 & 984 & 7 & $1105^{a}$ \\
\hline 9 & $1054^{a}$ & 10 & $1111^{\mathrm{a}}$ \\
\hline 12 & $1054^{a}$ & 11 & $1130^{a}$ \\
\hline 13 & 977 & & \\
\hline 14 & $1054^{\mathrm{a}}$ & & \\
\hline 15 & 1024 & & \\
\hline 16 & $1087^{a}$ & & \\
\hline 17 & $1058^{a}$ & & \\
\hline
\end{tabular}

$a_{\text {Used to calculate }} \mathrm{v}_{50}$ ballistic limit. 
Sample 10 (moderate wear); tested dry

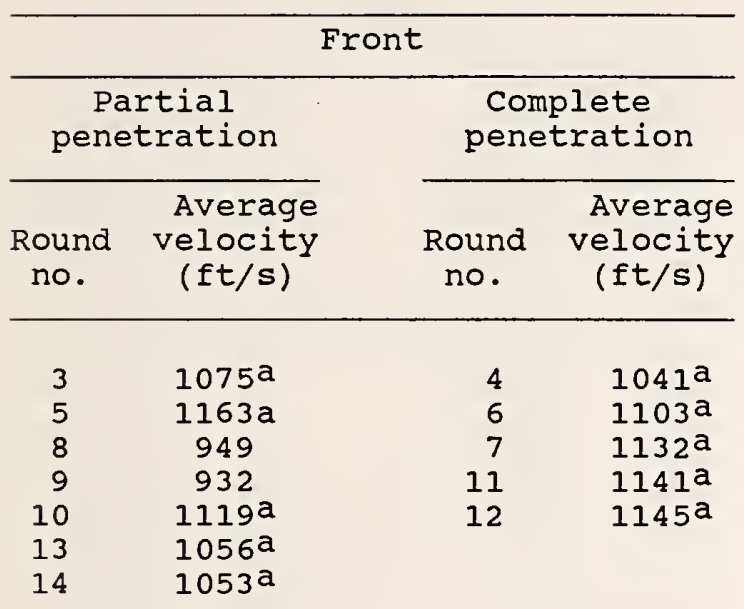

\begin{tabular}{|c|c|c|c|}
\hline \multicolumn{4}{|c|}{ Back } \\
\hline \multicolumn{2}{|c|}{$\begin{array}{c}\text { Partial } \\
\text { penetration }\end{array}$} & \multicolumn{2}{|c|}{$\begin{array}{c}\text { Complete } \\
\text { penetration }\end{array}$} \\
\hline $\begin{array}{c}\text { Round } \\
\text { no. }\end{array}$ & $\begin{array}{c}\text { Average } \\
\text { velocity } \\
(\mathrm{ft} / \mathrm{s})\end{array}$ & $\begin{array}{c}\text { Round } \\
\text { no. }\end{array}$ & $\begin{array}{c}\text { Average } \\
\text { velocity } \\
\text { (ft/s) }\end{array}$ \\
\hline 5 & 1077 & 7 & 1277 \\
\hline 6 & 1073 & 8 & $1160^{a}$ \\
\hline 10 & 974 & 9 & $1208^{a}$ \\
\hline 11 & $1136^{a}$ & 12 & $1210^{a}$ \\
\hline 13 & $1158^{a}$ & 15 & $1210^{a}$ \\
\hline 14 & $1113^{a}$ & 18 & $1101^{\mathrm{a}}$ \\
\hline 16 & 1136 a & & \\
\hline 17 & 1070 & & \\
\hline 19 & $1220^{a}$ & & \\
\hline
\end{tabular}

Sample 12 (Light wear); tested wet

\begin{tabular}{|c|c|c|c|}
\hline \multicolumn{4}{|c|}{ Front } \\
\hline \multicolumn{2}{|c|}{$\begin{array}{c}\text { Partial } \\
\text { penetration }\end{array}$} & \multicolumn{2}{|c|}{$\begin{array}{l}\text { Complete } \\
\text { penetration }\end{array}$} \\
\hline $\begin{array}{l}\text { Round } \\
\text { no. }\end{array}$ & $\begin{array}{c}\text { Average } \\
\text { velocity } \\
\text { (ft/s) }\end{array}$ & $\begin{array}{l}\text { Round } \\
\text { no. }\end{array}$ & $\begin{array}{c}\text { Average } \\
\text { velocity } \\
\text { (ft/s) }\end{array}$ \\
\hline 10 & $1070^{a}$ & 2 & $1184^{a}$ \\
\hline 11 & $1062^{a}$ & 9 & $1174^{a}$ \\
\hline 12 & $1113^{a}$ & 15 & $1163^{a}$ \\
\hline 13 & $1107^{a}$ & 16 & 1192 \\
\hline 14 & $1111^{a}$ & 17 & $1113^{a}$ \\
\hline & & 18 & $1165^{a}$ \\
\hline
\end{tabular}

\begin{tabular}{|c|c|c|c|}
\hline \multicolumn{4}{|c|}{ Back } \\
\hline \multicolumn{2}{|c|}{$\begin{array}{c}\text { Partial } \\
\text { penetration }\end{array}$} & \multicolumn{2}{|c|}{$\begin{array}{c}\text { Complete } \\
\text { penetration }\end{array}$} \\
\hline $\begin{array}{l}\text { Round } \\
\text { no. }\end{array}$ & $\begin{array}{c}\text { Average } \\
\text { velocity } \\
\text { (ft/s) }\end{array}$ & $\begin{array}{l}\text { Round } \\
\text { no. }\end{array}$ & $\begin{array}{c}\text { Average } \\
\text { velocity } \\
(\mathrm{ft} / \mathrm{s})\end{array}$ \\
\hline 3 & 861 & 6 & $1134^{\mathrm{a}}$ \\
\hline 4 & $1089^{a}$ & 8 & $1165^{a}$ \\
\hline 5 & 1040 & 10 & $1095 \mathrm{a}$ \\
\hline 7 & $1062^{a}$ & 14 & $1160^{a}$ \\
\hline 9 & $1097^{a}$ & 16 & 1192 \\
\hline 11 & 982 & 17 & $1138 \mathrm{a}$ \\
\hline 12 & $1085^{a}$ & & \\
\hline 13 & $1097^{a}$ & & \\
\hline 15 & 964 & & \\
\hline
\end{tabular}

Used to calculate $v_{50}$ ballistic limit. 
Sample 13 (heavy wear); tested wet

\begin{tabular}{|c|c|c|c|}
\hline \multicolumn{4}{|c|}{ Front } \\
\hline \multicolumn{2}{|c|}{$\begin{array}{c}\text { Partial } \\
\text { penetration }\end{array}$} & \multicolumn{2}{|c|}{$\begin{array}{l}\text { Complete } \\
\text { penetration }\end{array}$} \\
\hline $\begin{array}{l}\text { Round } \\
\text { no. }\end{array}$ & $\begin{array}{c}\text { Average } \\
\text { velocity } \\
\text { (ft/s) }\end{array}$ & $\begin{array}{l}\text { Round } \\
\text { no. }\end{array}$ & $\begin{array}{c}\text { Average } \\
\text { velocity } \\
\text { (ft/s) }\end{array}$ \\
\hline 5 & $1103^{a}$ & 4 & $1158^{a}$ \\
\hline 7 & $1124 a$ & 6 & $1186^{a}$ \\
\hline 8 & 871 & 9 & 1165 \\
\hline 12 & 1064 & 11 & $1165^{a}$ \\
\hline 13 & 978 & 16 & 1235 \\
\hline 14 & $1147 \mathrm{a}$ & 17 & $1170^{a}$ \\
\hline 15 & 1087 & 19 & 1215 \\
\hline 18 & $1222^{a}$ & & \\
\hline
\end{tabular}

\begin{tabular}{|c|c|c|c|}
\hline \multicolumn{4}{|c|}{ Back } \\
\hline $\begin{array}{r}\mathrm{Pa} \\
\text { pene }\end{array}$ & $\begin{array}{l}\text { rtial } \\
\text { tration }\end{array}$ & $\begin{array}{l}\text { Cor } \\
\text { pen }\end{array}$ & $\begin{array}{l}\text { olete } \\
\text { tration }\end{array}$ \\
\hline $\begin{array}{l}\text { Round } \\
\text { no. }\end{array}$ & $\begin{array}{c}\text { Average } \\
\text { velocity } \\
\text { (ft/s) }\end{array}$ & $\begin{array}{l}\text { Round } \\
\text { no. }\end{array}$ & $\begin{array}{c}\text { Average } \\
\text { velocity } \\
\text { (ft/s) }\end{array}$ \\
\hline $\begin{array}{r}5 \\
6 \\
11 \\
14 \\
17\end{array}$ & $\begin{array}{l}1210^{a} \\
1130^{a} \\
1085^{a} \\
1128^{a} \\
1073\end{array}$ & $\begin{array}{r}3 \\
4 \\
7 \\
8 \\
9 \\
10 \\
12 \\
13 \\
15 \\
18 \\
19 \\
20\end{array}$ & $\begin{array}{l}1143 \\
1103 a \\
1126 a \\
1172 \\
1138 \\
1163 \\
1156 \\
1132 a \\
1117 a \\
1186 \\
1143 \\
1174\end{array}$ \\
\hline
\end{tabular}

Sample 17 (heavy wear); tested dry

\begin{tabular}{|c|c|c|c|}
\hline \multicolumn{4}{|c|}{ Front } \\
\hline \multicolumn{2}{|c|}{$\begin{array}{c}\text { Partial } \\
\text { penetration }\end{array}$} & \multicolumn{2}{|c|}{$\begin{array}{l}\text { Complete } \\
\text { penetration }\end{array}$} \\
\hline $\begin{array}{c}\text { Round } \\
\text { no. }\end{array}$ & $\begin{array}{c}\text { Average } \\
\text { velocity } \\
(\mathrm{ft} / \mathrm{s})\end{array}$ & $\begin{array}{c}\text { Round } \\
\text { no. }\end{array}$ & $\begin{array}{c}\text { Average } \\
\text { velocity } \\
\text { (ft/s) }\end{array}$ \\
\hline 3 & $1089^{a}$ & 7 & 1285 \\
\hline 4 & $1119 a$ & 12 & 1232 \\
\hline 6 & $1132^{a}$ & 13 & $1192^{a}$ \\
\hline 8 & $1107 a$ & 14 & $1192^{a}$ \\
\hline 9 & 840 & 15 & 1215 \\
\hline 10 & 722 & 16 & $1154 \mathrm{a}$ \\
\hline 11 & 867 & 18 & $1186^{a}$ \\
\hline 17 & $1163^{a}$ & 20 & $1200^{a}$ \\
\hline 19 & 1071 & & \\
\hline
\end{tabular}

\begin{tabular}{|c|c|c|c|}
\hline \multicolumn{4}{|c|}{ Back } \\
\hline \multicolumn{2}{|c|}{$\begin{array}{c}\text { Partial } \\
\text { penetration }\end{array}$} & \multicolumn{2}{|c|}{$\begin{array}{l}\text { Complete } \\
\text { penetration }\end{array}$} \\
\hline $\begin{array}{l}\text { Round } \\
\text { no. }\end{array}$ & $\begin{array}{c}\text { Average } \\
\text { velocity } \\
\text { (ft/s) }\end{array}$ & $\begin{array}{l}\text { Round } \\
\text { no. }\end{array}$ & $\begin{array}{c}\text { Average } \\
\text { velocity } \\
\text { (ft/s) }\end{array}$ \\
\hline 6 & $1107 \mathrm{a}$ & 3 & 1266 \\
\hline 11 & $1040^{a}$ & 4 & 1245 \\
\hline 13 & 1007 & 5 & 1220 \\
\hline 14 & $1058 \mathrm{a}$ & 7 & 1210 \\
\hline 17 & $1022^{a}$ & 8 & $1083^{a}$ \\
\hline 18 & $1079^{a}$ & 9 & 1220 \\
\hline \multirow[t]{5}{*}{19} & 1003 & 10 & 1149 \\
\hline & & 12 & $1103^{2}$ \\
\hline & & 15 & $1130^{a}$ \\
\hline & & 16 & $1068^{a}$ \\
\hline & & 20 & $1062^{a}$ \\
\hline
\end{tabular}

$a_{\text {Used to }}$ calculate $v_{50}$ ballistic limit. 
Sample 18 (heavy wear); tested wet

\begin{tabular}{|c|c|c|c|}
\hline \multicolumn{4}{|c|}{ Front } \\
\hline \multicolumn{2}{|c|}{$\begin{array}{c}\text { Partial } \\
\text { penetration }\end{array}$} & \multicolumn{2}{|c|}{$\begin{array}{l}\text { Complete } \\
\text { penetration }\end{array}$} \\
\hline $\begin{array}{c}\text { Round } \\
\text { no. }\end{array}$ & $\begin{array}{c}\text { Average } \\
\text { velocity } \\
\text { (ft/s) }\end{array}$ & $\begin{array}{l}\text { Round } \\
\text { no. }\end{array}$ & $\begin{array}{c}\text { Average } \\
\text { velocity } \\
\text { (ft/s) }\end{array}$ \\
\hline 5 & $1097^{a}$ & 3 & $1109^{a}$ \\
\hline 6 & $1034^{a}$ & 4 & $1122^{a}$ \\
\hline 9 & $1031^{a}$ & 7 & $1130^{a}$ \\
\hline 11 & 997 & 8 & $1099^{a}$ \\
\hline 12 & $1020^{a}$ & 10 & $1014^{a}$ \\
\hline 14 & $1089^{a}$ & 13 & 1154 \\
\hline
\end{tabular}

\begin{tabular}{|c|c|c|c|}
\hline \multicolumn{4}{|c|}{ Back } \\
\hline \multicolumn{2}{|c|}{$\begin{array}{c}\text { Partial } \\
\text { penetration }\end{array}$} & \multicolumn{2}{|c|}{$\begin{array}{l}\text { Complete } \\
\text { penetration }\end{array}$} \\
\hline $\begin{array}{l}\text { Round } \\
\text { no. }\end{array}$ & $\begin{array}{c}\text { Average } \\
\text { velocity } \\
\text { (ft/s) }\end{array}$ & $\begin{array}{l}\text { Round } \\
\text { no. }\end{array}$ & $\begin{array}{c}\text { Average } \\
\text { velocity } \\
\text { (ft/s) }\end{array}$ \\
\hline 3 & $1060^{a}$ & 5 & $1079^{a}$ \\
\hline 4 & $1066^{a}$ & 6 & $1097^{a}$ \\
\hline 7 & $1008^{a}$ & 8 & $1070^{a}$ \\
\hline 10 & $1097^{a}$ & 9 & $1062^{a}$ \\
\hline 11 & $1073^{a}$ & 13 & $1130^{a}$ \\
\hline 12 & 995 & & \\
\hline
\end{tabular}

Sample 22 (unused); tested wet

\begin{tabular}{|c|c|c|c|}
\hline \multicolumn{4}{|c|}{ Front } \\
\hline \multicolumn{2}{|c|}{$\begin{array}{c}\text { Partial } \\
\text { penetration }\end{array}$} & \multicolumn{2}{|c|}{$\begin{array}{l}\text { Complete } \\
\text { penetration }\end{array}$} \\
\hline $\begin{array}{l}\text { Round } \\
\text { no. }\end{array}$ & $\begin{array}{c}\text { Average } \\
\text { velocity } \\
(\mathrm{ft} / \mathrm{s})\end{array}$ & $\begin{array}{l}\text { Round } \\
\text { no. }\end{array}$ & $\begin{array}{c}\text { Average } \\
\text { velocity } \\
\text { (ft/s) }\end{array}$ \\
\hline 3 & 1073 & 6 & 1261 \\
\hline 4 & 1047 & 8 & $1176^{\mathrm{a}}$ \\
\hline 5 & $1103^{a}$ & 12 & $1120^{a}$ \\
\hline 7 & 992 & 13 & $1212^{a}$ \\
\hline 9 & $1132^{\mathrm{a}}$ & 14 & $1227^{a}$ \\
\hline 10 & 934 & 19 & $1192^{a}$ \\
\hline 11 & 1079 & & \\
\hline 15 & 1085 & & \\
\hline 16 & $1132^{\mathrm{a}}$ & & \\
\hline 17 & 1089 & & \\
\hline 18 & $1117^{a}$ & & \\
\hline 20 & $1147^{a}$ & & \\
\hline
\end{tabular}

\begin{tabular}{|c|c|c|c|}
\hline \multicolumn{4}{|c|}{ Back } \\
\hline \multicolumn{2}{|c|}{$\begin{array}{c}\text { Partial } \\
\text { penetration }\end{array}$} & \multicolumn{2}{|c|}{$\begin{array}{c}\text { Complete } \\
\text { penetration }\end{array}$} \\
\hline $\begin{array}{l}\text { Round } \\
\text { no. }\end{array}$ & $\begin{array}{c}\text { Average } \\
\text { velocity } \\
\text { (ft/s) }\end{array}$ & $\begin{array}{l}\text { Round } \\
\text { no. }\end{array}$ & $\begin{array}{c}\text { Average } \\
\text { velocity } \\
\text { (ft/s) }\end{array}$ \\
\hline 3 & $1156^{\mathrm{a}}$ & 5 & $1192^{\mathrm{a}}$ \\
\hline 4 & 1077 & 7 & $1261^{a}$ \\
\hline 6 & $1195^{a}$ & 10 & $1224^{a}$ \\
\hline 8 & 1058 & 11 & $1149^{a}$ \\
\hline 9 & $1238^{a}$ & 15 & $1218^{a}$ \\
\hline 12 & 1099 & & \\
\hline 13 & $1149^{a}$ & & \\
\hline 14 & 1111 & & \\
\hline 16 & $1149^{a}$ & & \\
\hline
\end{tabular}

ased to calculate $v_{50}$ ballistic limit. 
Sample 23/24 (unused); tested dry

\begin{tabular}{|c|c|c|c|}
\hline \multicolumn{4}{|c|}{ Front (23) } \\
\hline \multicolumn{2}{|c|}{$\begin{array}{c}\text { Partial } \\
\text { penetration }\end{array}$} & \multicolumn{2}{|c|}{$\begin{array}{l}\text { Complete } \\
\text { penetration }\end{array}$} \\
\hline $\begin{array}{c}\text { Round } \\
\text { no. }\end{array}$ & $\begin{array}{c}\text { Average } \\
\text { velocity } \\
(\mathrm{ft} / \mathrm{s})\end{array}$ & $\begin{array}{l}\text { Round } \\
\text { no. }\end{array}$ & $\begin{array}{c}\text { Average } \\
\text { velocity } \\
(f t / s)\end{array}$ \\
\hline 3 & 950 & 4 & 1255 \\
\hline 9 & $1062 a$ & 5 & \\
\hline 10 & 958 & 6 & $1034^{a}$ \\
\hline 11 & $1045^{a}$ & 7 & 1122 \\
\hline 14 & $1007^{a}$ & 8 & $988^{a}$ \\
\hline 16 & 948 & 12 & $1047^{a}$ \\
\hline 17 & $992^{a}$ & 13 & $1002^{a}$ \\
\hline 18 & $1083^{a}$ & 15 & $1095^{a}$ \\
\hline
\end{tabular}

\begin{tabular}{|c|c|c|c|}
\hline \multicolumn{4}{|c|}{ Back (24) } \\
\hline \multicolumn{2}{|c|}{$\begin{array}{c}\text { Partial } \\
\text { penetration }\end{array}$} & \multicolumn{2}{|c|}{$\begin{array}{l}\text { Complete } \\
\text { penetration }\end{array}$} \\
\hline $\begin{array}{l}\text { Round } \\
\text { no. }\end{array}$ & $\begin{array}{c}\text { Average } \\
\text { velocity } \\
(\mathrm{ft} / \mathrm{s})\end{array}$ & $\begin{array}{l}\text { Round } \\
\text { no. }\end{array}$ & $\begin{array}{c}\text { Average } \\
\text { velocity } \\
(\mathrm{ft} / \mathrm{s})\end{array}$ \\
\hline 4 & $1017^{a}$ & 3 & 1117 \\
\hline 5 & $1029^{a}$ & 7 & $1036^{a}$ \\
\hline 6 & 971 & 8 & $1049 a$ \\
\hline 9 & 970 & 10 & $1045^{a}$ \\
\hline 14 & 965 & 11 & $1083^{a}$ \\
\hline 16 & $1053^{a}$ & 12 & $1107^{a}$ \\
\hline 17 & $1079^{a}$ & 13 & 1156 \\
\hline 18 & $1005^{a}$ & 15 & 1245 \\
\hline
\end{tabular}

Sample 30' (medium wear); tested wet

\begin{tabular}{|c|c|c|c|}
\hline \multicolumn{4}{|c|}{ Front } \\
\hline $\begin{array}{r}P c \\
\text { pene }\end{array}$ & $\begin{array}{l}\text { rtial } \\
\text { tration }\end{array}$ & $\begin{array}{l}\text { Con } \\
\text { pene }\end{array}$ & $\begin{array}{l}\text { plete } \\
\text { tration }\end{array}$ \\
\hline $\begin{array}{c}\text { Round } \\
\text { no. }\end{array}$ & $\begin{array}{c}\text { Average } \\
\text { velocity } \\
\text { (ft/s) }\end{array}$ & $\begin{array}{c}\text { Round } \\
\text { no. }\end{array}$ & $\begin{array}{c}\text { Average } \\
\text { velocity } \\
\text { (ft/s) }\end{array}$ \\
\hline $\begin{array}{r}5 \\
6 \\
8 \\
9 \\
14 \\
17\end{array}$ & $\begin{array}{c}1081^{a} \\
1105^{a} \\
1163^{a} \\
1165^{a} \\
940 \\
1083^{a}\end{array}$ & $\begin{array}{r}3 \\
4 \\
7 \\
10 \\
11 \\
12 \\
13 \\
15 \\
16\end{array}$ & $\begin{array}{l}1113^{a} \\
1156 \\
1081 \\
1230 \\
1184 \\
1138 a \\
1093^{2} \\
1152^{a} \\
1105 a\end{array}$ \\
\hline
\end{tabular}

\begin{tabular}{|c|c|c|c|}
\hline \multicolumn{4}{|c|}{ Back } \\
\hline \multicolumn{2}{|c|}{$\begin{array}{c}\text { Partial } \\
\text { penetration }\end{array}$} & \multicolumn{2}{|c|}{$\begin{array}{l}\text { Complete } \\
\text { penetration }\end{array}$} \\
\hline $\begin{array}{c}\text { Round } \\
\text { no. }\end{array}$ & $\begin{array}{c}\text { Average } \\
\text { velocity } \\
(\mathrm{ft} / \mathrm{s})\end{array}$ & $\begin{array}{l}\text { Round } \\
\text { no. }\end{array}$ & $\begin{array}{c}\text { Average } \\
\text { velocity } \\
(\mathrm{ft} / \mathrm{s})\end{array}$ \\
\hline 5 & $1134^{a}$ & 3 & $1134 a$ \\
\hline 7 & $1138^{a}$ & 4 & $1093^{a}$ \\
\hline 8 & 992 & 6 & 1085 \\
\hline 11 & $1128^{a}$ & 9 & 1210 \\
\hline 13 & $1073^{a}$ & 10 & $1130^{a}$ \\
\hline 14 & 982 & 12 & $1172^{a}$ \\
\hline 16 & 914 & 15 & $1176^{a}$ \\
\hline 17 & $1056^{a}$ & & \\
\hline
\end{tabular}

aused to calculate $v_{50}$ ballistic limit. 
Sample 31 (heavy wear); tested dry

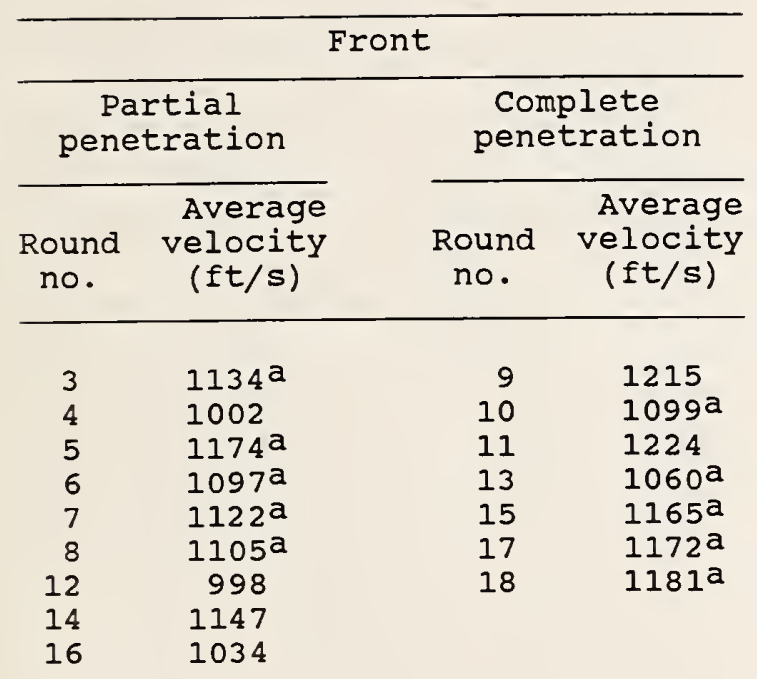

\begin{tabular}{|c|c|c|c|}
\hline \multicolumn{4}{|c|}{ Back } \\
\hline $\begin{array}{r}\mathrm{Pa} \\
\text { pene }\end{array}$ & $\begin{array}{l}\text { etial } \\
\text { eration }\end{array}$ & $\begin{array}{l}\text { Com } \\
\text { pene }\end{array}$ & $\begin{array}{l}\text { plete } \\
\text { tration }\end{array}$ \\
\hline $\begin{array}{l}\text { Round } \\
\text { no. }\end{array}$ & $\begin{array}{c}\text { Average } \\
\text { velocity } \\
\text { (ft/s) }\end{array}$ & $\begin{array}{c}\text { Round } \\
\text { no. }\end{array}$ & $\begin{array}{c}\text { Average } \\
\text { velocity } \\
\text { (ft/s) }\end{array}$ \\
\hline $\begin{array}{r}4 \\
5 \\
7 \\
10 \\
15 \\
16 \\
22\end{array}$ & $\begin{array}{c}1120 a \\
1093^{a} \\
1208 \mathrm{a} \\
958 \\
1075 \\
1154^{a} \\
1010\end{array}$ & $\begin{array}{r}6 \\
8 \\
9 \\
11 \\
12 \\
13 \\
14 \\
17 \\
18 \\
19 \\
20 \\
21\end{array}$ & $\begin{array}{l}1271 \\
1280 \\
1261 \\
1245 \\
1195 a \\
1181^{a} \\
1240 \\
1230 \\
1240 \\
1136^{a} \\
1132^{a} \\
1299\end{array}$ \\
\hline
\end{tabular}

Sample 33 (Light wear); tested dry

\begin{tabular}{|c|c|c|c|}
\hline \multicolumn{4}{|c|}{ Front } \\
\hline \multicolumn{2}{|c|}{$\begin{array}{c}\text { Partial } \\
\text { penetration }\end{array}$} & \multicolumn{2}{|c|}{$\begin{array}{l}\text { Complete } \\
\text { penetration }\end{array}$} \\
\hline $\begin{array}{c}\text { Round } \\
\text { no. }\end{array}$ & $\begin{array}{c}\text { Average } \\
\text { velocity } \\
\text { (ft/s) }\end{array}$ & $\begin{array}{l}\text { Round } \\
\text { no. }\end{array}$ & $\begin{array}{c}\text { Average } \\
\text { velocity } \\
\text { (ft/s) }\end{array}$ \\
\hline 3 & 977 & 6 & $1130^{a}$ \\
\hline 4 & $1068 a$ & 11 & $1091 a$ \\
\hline 5 & 1031 & 14 & $1124 a$ \\
\hline 7 & $1087^{a}$ & 15 & $1062^{a}$ \\
\hline 8 & $1181^{a}$ & 17 & $1138^{a}$ \\
\hline 9 & 861 & & \\
\hline 10 & $1056^{a}$ & & \\
\hline 12 & $1101 a$ & & \\
\hline 13 & 1005 & & \\
\hline 16 & 1027 & & \\
\hline
\end{tabular}

\begin{tabular}{|c|c|c|c|}
\hline \multicolumn{4}{|c|}{ Back } \\
\hline \multicolumn{2}{|c|}{$\begin{array}{c}\text { Partial } \\
\text { penetration }\end{array}$} & \multicolumn{2}{|c|}{$\begin{array}{l}\text { Complete } \\
\text { penetration }\end{array}$} \\
\hline $\begin{array}{c}\text { Round } \\
\text { no. }\end{array}$ & $\begin{array}{c}\text { Average } \\
\text { velocity } \\
\text { (ft/s) }\end{array}$ & $\begin{array}{l}\text { Round } \\
\text { no. }\end{array}$ & $\begin{array}{c}\text { Average } \\
\text { velocity } \\
\text { (ft/s) }\end{array}$ \\
\hline 3 & $1167 a$ & 7 & $1200^{a}$ \\
\hline 4 & 1056 & 8 & $1183^{a}$ \\
\hline 5 & 1073 & 10 & $1111^{a}$ \\
\hline 6 & $1122^{a}$ & 14 & 1220 \\
\hline 9 & 998 & 19 & $1152^{\mathrm{a}}$ \\
\hline 11 & 1042 & 22 & 1210 \\
\hline 12 & $1075^{a}$ & 23 & $1130^{a}$ \\
\hline 13 & 1064 & & \\
\hline 15 & 1031 & & \\
\hline 16 & 1012 & & \\
\hline 17 & 1044 & & \\
\hline 18 & $1126 a$ & & \\
\hline 20 & $1089 a$ & & \\
\hline 21 & 1053 & & \\
\hline
\end{tabular}

ased to calculate $v_{50}$ ballistic limit. 


$$
\begin{gathered}
\text { APPENDIX B } \\
\mathrm{V}_{50} \text { Ballistic Limit Test Data } \\
\text { Seven Layer Kevlar Soft Body Armor }
\end{gathered}
$$

22 Caliber, 40 Grain, Lead Round Nose Projectile 
Sample 2 (unused); tested dry

\begin{tabular}{|c|c|c|c|}
\hline \multicolumn{4}{|c|}{ Front } \\
\hline \multicolumn{2}{|c|}{$\begin{array}{c}\text { Partial } \\
\text { penetration }\end{array}$} & \multicolumn{2}{|c|}{$\begin{array}{l}\text { Complete } \\
\text { penetration }\end{array}$} \\
\hline $\begin{array}{c}\text { Round } \\
\text { no. }\end{array}$ & $\begin{array}{c}\text { Average } \\
\text { velocity } \\
(\mathrm{ft} / \mathrm{s})\end{array}$ & $\begin{array}{l}\text { Round } \\
\text { no. }\end{array}$ & $\begin{array}{l}\text { Average } \\
\text { velocity } \\
(\mathrm{ft} / \mathrm{s})\end{array}$ \\
\hline 3 & $1163^{a}$ & 4 & $1227 a$ \\
\hline 6 & 1170 & 5 & $1210^{a}$ \\
\hline 9 & $1111 a$ & 7 & $1186 a$ \\
\hline 10 & 1085 & 8 & $1165^{a}$ \\
\hline 11 & $1202^{a}$ & 12 & $1178^{a}$ \\
\hline 14 & $1178 a$ & 13 & 1190 \\
\hline
\end{tabular}

\begin{tabular}{ccccc}
\hline & \multicolumn{3}{c}{ Back } \\
\hline $\begin{array}{c}\text { Partial } \\
\text { penetration }\end{array}$ & & \multicolumn{2}{c}{$\begin{array}{c}\text { Complete } \\
\text { penetration }\end{array}$} \\
\cline { 5 - 6 } $\begin{array}{c}\text { Round } \\
\text { no. }\end{array}$ & $\begin{array}{c}\text { Average } \\
\text { velocity } \\
\text { (ft/s) }\end{array}$ & & $\begin{array}{c}\text { Round } \\
\text { no. }\end{array}$ & $\begin{array}{c}\text { Average } \\
\text { velocity } \\
\text { (ft/s) }\end{array}$ \\
\hline & & & & \\
3 & $1200^{a}$ & & 8 & 1316 \\
4 & $1170^{a}$ & & 10 & $1266^{a}$ \\
5 & 1224 & & 11 & $1222^{a}$ \\
6 & $1141^{a}$ & & 12 & $1188^{a}$ \\
7 & $1238^{a}$ & 14 & $1220^{a}$ \\
9 & 1310 & 15 & $1212^{a}$ \\
13 & $1176^{a}$ & &
\end{tabular}

Sample 3 (unused); tested wet

\begin{tabular}{|c|c|c|c|}
\hline \multicolumn{4}{|c|}{ Front } \\
\hline \multicolumn{2}{|c|}{$\begin{array}{c}\text { Partial } \\
\text { penetration }\end{array}$} & \multicolumn{2}{|c|}{$\begin{array}{l}\text { Complete } \\
\text { penetration }\end{array}$} \\
\hline $\begin{array}{c}\text { Round } \\
\text { no. }\end{array}$ & $\begin{array}{c}\text { Average } \\
\text { velocity } \\
(\mathrm{ft} / \mathrm{s})\end{array}$ & $\begin{array}{l}\text { Round } \\
\text { no. }\end{array}$ & $\begin{array}{c}\text { Average } \\
\text { velocity } \\
(\mathrm{ft} / \mathrm{s})\end{array}$ \\
\hline 3 & 1130 & 5 & $1208^{a}$ \\
\hline 4 & $1165^{a}$ & 6 & $1198 a$ \\
\hline 7 & $1143^{a}$ & 8 & $1158^{a}$ \\
\hline 9 & $1143^{a}$ & 10 & $1184^{a}$ \\
\hline 12 & $1176 \mathrm{a}$ & 11 & $1152^{a}$ \\
\hline 13 & $1165^{a}$ & & \\
\hline
\end{tabular}

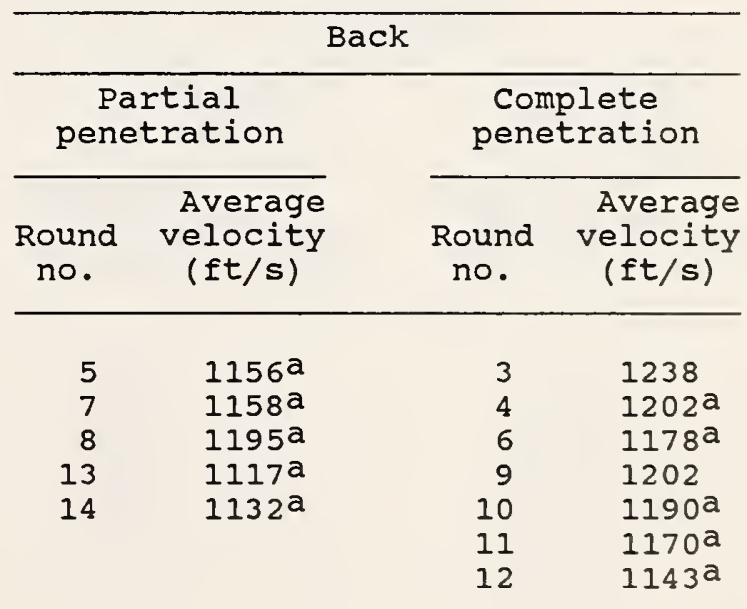

ased to calculate $v_{50}$ ballistic limit. 
Sample 3 (unused); retested dry

\begin{tabular}{|c|c|c|c|}
\hline \multicolumn{4}{|c|}{ Front } \\
\hline \multicolumn{2}{|c|}{$\begin{array}{c}\text { Partial } \\
\text { penetration }\end{array}$} & \multicolumn{2}{|c|}{$\begin{array}{l}\text { Complete } \\
\text { penetration }\end{array}$} \\
\hline $\begin{array}{l}\text { Round } \\
\text { no. }\end{array}$ & $\begin{array}{l}\text { Average } \\
\text { velocity } \\
\text { (ft/s) }\end{array}$ & $\begin{array}{l}\text { Round } \\
\text { no. }\end{array}$ & $\begin{array}{c}\text { Average } \\
\text { velocity } \\
\text { (ft/s) }\end{array}$ \\
\hline 16 & 1017 & 14 & $1176^{a}$ \\
\hline 17 & $1068 a$ & 15 & $1145^{a}$ \\
\hline 18 & $1066^{a}$ & 19 & $1154^{a}$ \\
\hline 22 & $1070^{a}$ & 20 & $1109 a$ \\
\hline 23 & $1066^{a}$ & 21 & $1085^{a}$ \\
\hline 24 & $1085^{a}$ & & \\
\hline
\end{tabular}

\begin{tabular}{|c|c|c|c|}
\hline \multicolumn{4}{|c|}{ Back } \\
\hline \multicolumn{2}{|c|}{$\begin{array}{c}\text { Partial } \\
\text { penetration }\end{array}$} & \multicolumn{2}{|c|}{$\begin{array}{l}\text { Complete } \\
\text { penetration }\end{array}$} \\
\hline $\begin{array}{c}\text { Round } \\
\text { no. }\end{array}$ & $\begin{array}{l}\text { Average } \\
\text { velocity } \\
\text { (ft/s) }\end{array}$ & $\begin{array}{c}\text { Round } \\
\text { no. }\end{array}$ & $\begin{array}{c}\text { Average } \\
\text { velocity } \\
\text { (ft/s) }\end{array}$ \\
\hline 15 & $1158^{a}$ & 16 & $1202^{a}$ \\
\hline 17 & $1163 a$ & 18 & 1280 \\
\hline 19 & $1181^{a}$ & 21 & $1232^{\mathrm{a}}$ \\
\hline 20 & $1220^{a}$ & 22 & $1220^{a}$ \\
\hline \multirow[t]{2}{*}{26} & $1117 a$ & 23 & $1218^{a}$ \\
\hline & & 25 & $1170^{\mathrm{a}}$ \\
\hline
\end{tabular}

Sample 6 (heavy wear); tested wet

\begin{tabular}{|c|c|c|c|}
\hline \multicolumn{4}{|c|}{ Front } \\
\hline \multicolumn{2}{|c|}{$\begin{array}{c}\text { Partial } \\
\text { penetration }\end{array}$} & \multicolumn{2}{|c|}{$\begin{array}{l}\text { Complete } \\
\text { penetration }\end{array}$} \\
\hline $\begin{array}{l}\text { Round } \\
\text { no. }\end{array}$ & $\begin{array}{c}\text { Average } \\
\text { velocity } \\
(\mathrm{ft} / \mathrm{s})\end{array}$ & $\begin{array}{l}\text { Round } \\
\text { no. }\end{array}$ & $\begin{array}{l}\text { Average } \\
\text { velocity } \\
\text { (ft/s) }\end{array}$ \\
\hline 3 & 1024 & 1 & $1054^{a}$ \\
\hline 4 & 1007 & 2 & $1049 \mathrm{a}$ \\
\hline 5 & $1083 a$ & 12 & $1089 a$ \\
\hline 6 & $1075 a$ & 14 & $1109 a$ \\
\hline 7 & $1091 a$ & 15 & $1060^{a}$ \\
\hline 8 & 1051 & & \\
\hline 9 & $1070^{a}$ & & \\
\hline 11 & $1066^{a}$ & & \\
\hline 13 & 1062 & & \\
\hline
\end{tabular}

\begin{tabular}{|c|c|c|c|}
\hline \multicolumn{4}{|c|}{ Back } \\
\hline \multicolumn{2}{|c|}{$\begin{array}{c}\text { Partial } \\
\text { penetration }\end{array}$} & \multicolumn{2}{|c|}{$\begin{array}{l}\text { Complete } \\
\text { penetration }\end{array}$} \\
\hline $\begin{array}{l}\text { Round } \\
\text { no. }\end{array}$ & $\begin{array}{c}\text { Average } \\
\text { velocity } \\
(\mathrm{ft} / \mathrm{s})\end{array}$ & $\begin{array}{l}\text { Round } \\
\text { no. }\end{array}$ & $\begin{array}{c}\text { Average } \\
\text { velocity } \\
\text { (ft/s) }\end{array}$ \\
\hline 3 & 1099 & 5 & 1288 \\
\hline 4 & $1132^{a}$ & 6 & 1250 \\
\hline 7 & $1172^{a}$ & 9 & 1230 \\
\hline 8 & 1230 & 10 & $1202^{a}$ \\
\hline 15 & 1051 & 11 & 1208 \\
\hline 17 & $1113 a$ & 12 & 1208 \\
\hline 19 & 1081 & 13 & $1181^{a}$ \\
\hline \multirow[t]{2}{*}{20} & $1124^{a}$ & 14 & $1115^{\mathrm{a}}$ \\
\hline & & 18 & $1122^{a}$ \\
\hline
\end{tabular}

Note: Shot 10, no velocity reading.

${ }^{a}$ Used to calculate $v_{50}$ ballistic limit. 
Sample 6 (heavy wear); retested dry

\begin{tabular}{|c|c|c|c|}
\hline \multicolumn{4}{|c|}{ Front } \\
\hline \multicolumn{2}{|c|}{$\begin{array}{c}\text { Partial } \\
\text { penetration }\end{array}$} & \multicolumn{2}{|c|}{$\begin{array}{l}\text { Complete } \\
\text { penetration }\end{array}$} \\
\hline $\begin{array}{l}\text { Round } \\
\text { no. }\end{array}$ & $\begin{array}{c}\text { Average } \\
\text { velocity } \\
\text { (ft/s) }\end{array}$ & $\begin{array}{l}\text { Round } \\
\text { no. }\end{array}$ & $\begin{array}{c}\text { Average } \\
\text { velocity } \\
\text { (ft/s) }\end{array}$ \\
\hline 16 & 1099 & 17 & $1158 \mathrm{a}$ \\
\hline 18 & $1170^{a}$ & 19 & $1240^{a}$ \\
\hline 20 & $1149 a$ & 22 & $1190^{a}$ \\
\hline 21 & $1145^{a}$ & 24 & $1205^{a}$ \\
\hline 23 & $1167^{a}$ & 25 & $1208^{a}$ \\
\hline 26 & $1192^{a}$ & & \\
\hline
\end{tabular}

\begin{tabular}{|c|c|c|c|}
\hline \multicolumn{4}{|c|}{ Back } \\
\hline \multicolumn{2}{|c|}{$\begin{array}{c}\text { Partial } \\
\text { penetration }\end{array}$} & \multicolumn{2}{|c|}{$\begin{array}{l}\text { Complete } \\
\text { penetration }\end{array}$} \\
\hline $\begin{array}{l}\text { Round } \\
\text { no. }\end{array}$ & $\begin{array}{l}\text { Average } \\
\text { velocity } \\
\text { (ft/s) }\end{array}$ & $\begin{array}{l}\text { Round } \\
\text { no. }\end{array}$ & $\begin{array}{c}\text { Average } \\
\text { velocity } \\
(\mathrm{ft} / \mathrm{s})\end{array}$ \\
\hline 25 & 1040 & 21 & $1143^{a}$ \\
\hline 26 & 1062 & 22 & $1163^{a}$ \\
\hline 27 & $1085^{a}$ & 23 & 1202 \\
\hline 28 & $1068^{a}$ & 24 & $1178^{a}$ \\
\hline 29 & $1138^{a}$ & 31 & $1158^{a}$ \\
\hline 30 & $1165^{a}$ & & \\
\hline
\end{tabular}

Sample 7 (moderate wear); tested wet

\begin{tabular}{|c|c|c|c|}
\hline \multicolumn{4}{|c|}{ Front } \\
\hline $\begin{array}{r}\mathrm{Pa} \\
\text { pene }\end{array}$ & $\begin{array}{l}\text { tial } \\
\text { ration }\end{array}$ & $\begin{array}{r}\text { Con } \\
\text { pene }\end{array}$ & $\begin{array}{l}\text { olete } \\
\text { tration }\end{array}$ \\
\hline $\begin{array}{c}\text { Round } \\
\text { no. }\end{array}$ & $\begin{array}{c}\text { Average } \\
\text { velocity } \\
\text { (ft/s) }\end{array}$ & $\begin{array}{c}\text { Round } \\
\text { no. }\end{array}$ & $\begin{array}{c}\text { Average } \\
\text { velocity } \\
\text { (ft/s) }\end{array}$ \\
\hline $\begin{array}{r}9 \\
10 \\
11 \\
12 \\
14 \\
16 \\
18\end{array}$ & $\begin{array}{l}1071 \\
1143^{a} \\
1124 \\
1154^{a} \\
1152^{a} \\
1149 a \\
1156^{a}\end{array}$ & $\begin{array}{r}3 \\
4 \\
5 \\
6 \\
7 \\
8 \\
13 \\
15 \\
17\end{array}$ & $\begin{array}{l}1296 \\
1274 \\
1248 a \\
1235 a \\
1184 a \\
1154 a \\
1357 \\
1282 \\
1258 a\end{array}$ \\
\hline
\end{tabular}

\begin{tabular}{|c|c|c|c|}
\hline \multicolumn{4}{|c|}{ Back } \\
\hline \multicolumn{2}{|c|}{$\begin{array}{c}\text { Partial } \\
\text { penetration }\end{array}$} & \multicolumn{2}{|c|}{$\begin{array}{l}\text { Complete } \\
\text { penetration }\end{array}$} \\
\hline $\begin{array}{l}\text { Round } \\
\text { no. }\end{array}$ & $\begin{array}{l}\text { Average } \\
\text { velocity } \\
\text { (ft/s) }\end{array}$ & $\begin{array}{l}\text { Round } \\
\text { no. }\end{array}$ & $\begin{array}{c}\text { Average } \\
\text { velocity } \\
(\mathrm{ft} / \mathrm{s})\end{array}$ \\
\hline 6 & $1186^{a}$ & 3 & $1250^{a}$ \\
\hline 7 & $1200^{a}$ & 4 & $1230^{a}$ \\
\hline 8 & $1250^{a}$ & 5 & 1176 \\
\hline 9 & $1250^{a}$ & 11 & $1299 a$ \\
\hline \multirow[t]{2}{*}{10} & $1261^{a}$ & 12 & $1290^{a}$ \\
\hline & & 13 & $1293 a$ \\
\hline
\end{tabular}

ased to calculate $v_{50}$ ballistic limit. 
Sample 8 (heavy wear); tested dry

\begin{tabular}{|c|c|c|c|}
\hline \multicolumn{4}{|c|}{ Front } \\
\hline $\begin{array}{l}\mathrm{Pa} \\
\text { pene }\end{array}$ & $\begin{array}{l}\text { rtial } \\
\text { tration }\end{array}$ & $\begin{array}{l}\text { Com } \\
\text { pene }\end{array}$ & $\begin{array}{l}\text { lete } \\
\text { tration }\end{array}$ \\
\hline $\begin{array}{l}\text { Round } \\
\text { no. }\end{array}$ & $\begin{array}{l}\text { Average } \\
\text { velocity } \\
\text { (ft/s) }\end{array}$ & $\begin{array}{l}\text { Round } \\
\text { no. }\end{array}$ & $\begin{array}{l}\text { Average } \\
\text { velocity } \\
(\mathrm{ft} / \mathrm{s})\end{array}$ \\
\hline $\begin{array}{r}3 \\
4 \\
5 \\
6 \\
11\end{array}$ & $\begin{array}{l}1126^{a} \\
1134^{a} \\
1186^{a} \\
1215{ }^{a} \\
1222^{a}\end{array}$ & $\begin{array}{r}7 \\
8 \\
9 \\
10 \\
12 \\
13 \\
14\end{array}$ & $\begin{array}{l}1327 \\
1268 \\
1245 a \\
1232 \mathrm{a} \\
1245 \mathrm{a} \\
1220 \mathrm{a} \\
1176^{a}\end{array}$ \\
\hline
\end{tabular}

\begin{tabular}{|c|c|c|c|}
\hline \multicolumn{4}{|c|}{ Back } \\
\hline \multicolumn{2}{|c|}{$\begin{array}{c}\text { Partial } \\
\text { penetration }\end{array}$} & \multicolumn{2}{|c|}{$\begin{array}{l}\text { Complete } \\
\text { penetration }\end{array}$} \\
\hline $\begin{array}{c}\text { Round } \\
\text { no. }\end{array}$ & $\begin{array}{c}\text { Average } \\
\text { velocity } \\
(\mathrm{ft} / \mathrm{s})\end{array}$ & $\begin{array}{l}\text { Round } \\
\text { no. }\end{array}$ & $\begin{array}{l}\text { Average } \\
\text { velocity } \\
\text { (ft/s) }\end{array}$ \\
\hline 3 & $1200^{a}$ & 6 & $1205^{a}$ \\
\hline 4 & $1220^{a}$ & 7 & $1215 \mathrm{a}$ \\
\hline 5 & 1172 & 11 & $1215^{a}$ \\
\hline 8 & $1210^{a}$ & 12 & $1220^{a}$ \\
\hline 9 & $1181^{\mathrm{a}}$ & 13 & $1195^{a}$ \\
\hline 10 & $1186^{a}$ & & \\
\hline
\end{tabular}

Sample 9 (heavy wear); tested wet

\begin{tabular}{|c|c|c|c|}
\hline \multicolumn{4}{|c|}{ Front } \\
\hline $\begin{array}{r}P \\
\text { pen }\end{array}$ & $\begin{array}{l}\text { rtial } \\
\text { tration }\end{array}$ & $\begin{array}{l}\text { Cor } \\
\text { pene }\end{array}$ & $\begin{array}{l}\text { plete } \\
\text { tration }\end{array}$ \\
\hline $\begin{array}{c}\text { Round } \\
\text { no. }\end{array}$ & $\begin{array}{c}\text { Average } \\
\text { velocity } \\
(\mathrm{ft} / \mathrm{s})\end{array}$ & $\begin{array}{l}\text { Round } \\
\text { no. }\end{array}$ & $\begin{array}{c}\text { Average } \\
\text { velocity } \\
(\mathrm{ft} / \mathrm{s})\end{array}$ \\
\hline $\begin{array}{r}4 \\
7 \\
8 \\
10 \\
12\end{array}$ & $\begin{array}{l}1176^{a} \\
1158 a \\
1181 a \\
1174 a \\
1165 a\end{array}$ & $\begin{array}{r}3 \\
5 \\
6 \\
9 \\
11 \\
13 \\
14\end{array}$ & $\begin{array}{l}1154^{a} \\
1202^{a} \\
1165 \\
1170 a \\
1188^{a} \\
1202 \\
1258 a\end{array}$ \\
\hline
\end{tabular}

\begin{tabular}{|c|c|c|c|}
\hline \multicolumn{4}{|c|}{ Back } \\
\hline \multicolumn{2}{|c|}{$\begin{array}{c}\text { Partial } \\
\text { penetration }\end{array}$} & \multicolumn{2}{|c|}{$\begin{array}{l}\text { Complete } \\
\text { penetration }\end{array}$} \\
\hline $\begin{array}{l}\text { Round } \\
\text { no. }\end{array}$ & $\begin{array}{c}\text { Average } \\
\text { velocity } \\
(\mathrm{ft} / \mathrm{s})\end{array}$ & $\begin{array}{l}\text { Round } \\
\text { no. }\end{array}$ & $\begin{array}{l}\text { Average } \\
\text { velocity } \\
(\mathrm{ft} / \mathrm{s})\end{array}$ \\
\hline 4 & $1147^{a}$ & 3 & 1174 \\
\hline 9 & $1049 a$ & 5 & 1170 \\
\hline 11 & $1107 \mathrm{a}$ & 6 & 1145 \\
\hline 13 & $1101^{a}$ & 7 & $1113^{a}$ \\
\hline 15 & 1000 & 8 & 1105 \\
\hline \multirow[t]{4}{*}{16} & $1093^{a}$ & 10 & $1075^{\mathrm{a}}$ \\
\hline & & 12 & $1119^{a}$ \\
\hline & & 14 & $1085^{a}$ \\
\hline & & 16 & $1093^{\mathrm{a}}$ \\
\hline
\end{tabular}

Used to calculate $v_{50}$ ballistic limit. 
Sample 9 (heavy wear); retested dry

\begin{tabular}{|c|c|c|c|}
\hline \multicolumn{4}{|c|}{ Front } \\
\hline \multicolumn{2}{|c|}{$\begin{array}{c}\text { Partial } \\
\text { penetration }\end{array}$} & \multicolumn{2}{|c|}{$\begin{array}{l}\text { Complete } \\
\text { penetration }\end{array}$} \\
\hline $\begin{array}{c}\text { Round } \\
\text { no. }\end{array}$ & $\begin{array}{c}\text { Average } \\
\text { velocity } \\
\text { (ft/s) }\end{array}$ & $\begin{array}{l}\text { Round } \\
\text { no. }\end{array}$ & $\begin{array}{c}\text { Average } \\
\text { velocity } \\
\text { (ft/s) }\end{array}$ \\
\hline 16 & $1134^{\mathrm{a}}$ & 15 & $1188^{a}$ \\
\hline 17 & $1192^{\mathrm{a}}$ & 19 & $1215^{a}$ \\
\hline 18 & $1198^{a}$ & 20 & $1195^{a}$ \\
\hline 22 & $1149 a$ & 21 & $1160^{a}$ \\
\hline 23 & $1174^{a}$ & 24 & $1227^{a}$ \\
\hline
\end{tabular}

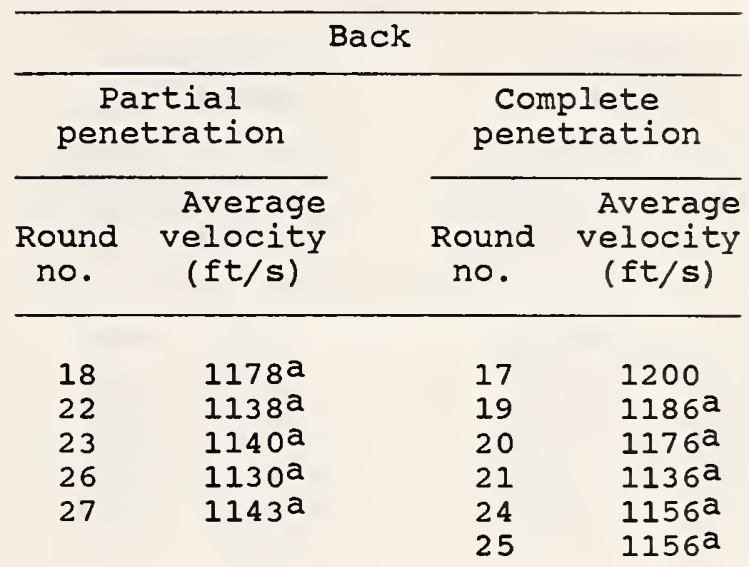

Sample 15 (Light wear); tested dry

\begin{tabular}{|c|c|c|c|}
\hline \multicolumn{4}{|c|}{ Front } \\
\hline \multicolumn{2}{|c|}{$\begin{array}{c}\text { Partial } \\
\text { penetration }\end{array}$} & \multicolumn{2}{|c|}{$\begin{array}{l}\text { Complete } \\
\text { penetration }\end{array}$} \\
\hline $\begin{array}{l}\text { Round } \\
\text { no. }\end{array}$ & $\begin{array}{c}\text { Average } \\
\text { velocity } \\
\text { (ft/s) }\end{array}$ & $\begin{array}{l}\text { Round } \\
\text { no. }\end{array}$ & $\begin{array}{c}\text { Average } \\
\text { velocity } \\
\text { (ft/s) }\end{array}$ \\
\hline 8 & 1128 & 4 & 1313 \\
\hline 9 & $1205^{a}$ & 5 & 1310 \\
\hline 10 & $1181^{\mathrm{a}}$ & 6 & 1277 \\
\hline 12 & 1186 & 7 & $1230^{\mathrm{a}}$ \\
\hline 13 & $1215^{a}$ & 11 & $1230^{a}$ \\
\hline 15 & $1190^{\mathrm{a}}$ & 14 & $1261^{a}$ \\
\hline \multirow[t]{3}{*}{19} & $1220^{a}$ & 16 & 1277 \\
\hline & & 17 & $1220^{a}$ \\
\hline & & 18 & $1240^{a}$ \\
\hline
\end{tabular}

\begin{tabular}{|c|c|c|c|}
\hline \multicolumn{4}{|c|}{ Back } \\
\hline \multicolumn{2}{|c|}{$\begin{array}{c}\text { Partial } \\
\text { penetration }\end{array}$} & \multicolumn{2}{|c|}{$\begin{array}{l}\text { Complete } \\
\text { penetration }\end{array}$} \\
\hline $\begin{array}{c}\text { Round } \\
\text { no. }\end{array}$ & $\begin{array}{c}\text { Average } \\
\text { velocity } \\
(\mathrm{ft} / \mathrm{s})\end{array}$ & $\begin{array}{c}\text { Round } \\
\text { no. }\end{array}$ & $\begin{array}{c}\text { Average } \\
\text { velocity } \\
(\mathrm{ft} / \mathrm{s})\end{array}$ \\
\hline 3 & $1186^{a}$ & 5 & 1285 \\
\hline 4 & $1202^{a}$ & 6 & 1364 \\
\hline 7 & $1280^{a}$ & 8 & $1310^{a}$ \\
\hline 10 & $1290^{a}$ & 9 & $1299 \mathrm{a}$ \\
\hline 14 & $1245^{a}$ & 11 & 1333 \\
\hline & & 12 & $1261^{a}$ \\
\hline & & 13 & $1304^{a}$ \\
\hline & & 15 & $1293^{a}$ \\
\hline
\end{tabular}

ased to calculate $v_{50}$ ballistic limit. 
Sample 20 (heavy wear); tested dry

\begin{tabular}{|c|c|c|c|}
\hline \multicolumn{4}{|c|}{ Front } \\
\hline \multicolumn{2}{|c|}{$\begin{array}{c}\text { Partial } \\
\text { penetration }\end{array}$} & \multicolumn{2}{|c|}{$\begin{array}{c}\text { Complete } \\
\text { penetration }\end{array}$} \\
\hline $\begin{array}{l}\text { Round } \\
\text { no. }\end{array}$ & $\begin{array}{c}\text { Average } \\
\text { velocity } \\
\text { (ft/s) }\end{array}$ & $\begin{array}{c}\text { Round } \\
\text { no. }\end{array}$ & $\begin{array}{c}\text { Average } \\
\text { velocity } \\
\text { (ft/s) }\end{array}$ \\
\hline 3 & 1220 & 4 & 1232 \\
\hline 8 & $1109^{a}$ & 5 & $1232^{a}$ \\
\hline 9 & 1149 & 6 & $1208^{a}$ \\
\hline 10 & 1126 & 7 & $1145^{\mathrm{a}}$ \\
\hline 12 & $1134^{\mathrm{a}}$ & 11 & $1160^{\mathrm{a}}$ \\
\hline 13 & $1134^{\mathrm{a}}$ & 14 & $1208^{a}$ \\
\hline 15 & $1147^{\mathrm{a}}$ & & \\
\hline
\end{tabular}

\begin{tabular}{|c|c|c|c|}
\hline \multicolumn{4}{|c|}{ Back } \\
\hline \multicolumn{2}{|c|}{$\begin{array}{c}\text { Partial } \\
\text { penetration }\end{array}$} & \multicolumn{2}{|c|}{$\begin{array}{l}\text { Complete } \\
\text { penetration }\end{array}$} \\
\hline $\begin{array}{l}\text { Round } \\
\text { no. }\end{array}$ & $\begin{array}{c}\text { Average } \\
\text { velocity } \\
(\mathrm{ft} / \mathrm{s})\end{array}$ & $\begin{array}{c}\text { Round } \\
\text { no. }\end{array}$ & $\begin{array}{c}\text { Average } \\
\text { velocity } \\
\text { (ft/s) }\end{array}$ \\
\hline 4 & $1147^{\mathrm{a}}$ & 3 & $1174^{\mathrm{a}}$ \\
\hline 5 & $1174^{\mathrm{a}}$ & 6 & $1178^{a}$ \\
\hline 8 & $1160^{\mathrm{a}}$ & 7 & $1195^{a}$ \\
\hline 9 & $1170^{\mathrm{a}}$ & 10 & $1198^{a}$ \\
\hline 12 & $1147^{a}$ & 11 & $1172^{a}$ \\
\hline
\end{tabular}

Sample 21 (light wear); tested wet

\begin{tabular}{|c|c|c|c|}
\hline \multicolumn{4}{|c|}{ Front } \\
\hline \multicolumn{2}{|c|}{$\begin{array}{c}\text { Partial } \\
\text { penetration }\end{array}$} & \multicolumn{2}{|c|}{$\begin{array}{l}\text { Complete } \\
\text { penetration }\end{array}$} \\
\hline $\begin{array}{c}\text { Round } \\
\text { no. }\end{array}$ & $\begin{array}{c}\text { Average } \\
\text { velocity } \\
\text { (ft/s) }\end{array}$ & $\begin{array}{l}\text { Round } \\
\text { no. }\end{array}$ & $\begin{array}{c}\text { Average } \\
\text { velocity } \\
(\mathrm{ft} / \mathrm{s})\end{array}$ \\
\hline 3 & 1077 & 6 & $1242^{\mathrm{a}}$ \\
\hline 4 & $1160^{a}$ & 10 & $1232^{\mathrm{a}}$ \\
\hline 5 & $1232^{\mathrm{a}}$ & 11 & $1248^{a}$ \\
\hline 7 & 1222 & 12 & 1252 \\
\hline 8 & 1235 & 13 & $1222^{a}$ \\
\hline 9 & $1242^{a}$ & 14 & $1198^{a}$ \\
\hline 15 & $1174^{\mathrm{a}}$ & & \\
\hline 16 & $1188^{a}$ & & \\
\hline
\end{tabular}

\begin{tabular}{|c|c|c|c|}
\hline \multicolumn{4}{|c|}{ Back } \\
\hline \multicolumn{2}{|c|}{$\begin{array}{c}\text { Partial } \\
\text { penetration }\end{array}$} & \multicolumn{2}{|c|}{$\begin{array}{l}\text { Complete } \\
\text { penetration }\end{array}$} \\
\hline $\begin{array}{l}\text { Round } \\
\text { no. }\end{array}$ & $\begin{array}{c}\text { Average } \\
\text { velocity } \\
(\mathrm{ft} / \mathrm{s})\end{array}$ & $\begin{array}{l}\text { Round } \\
\text { no. }\end{array}$ & $\begin{array}{c}\text { Average } \\
\text { velocity } \\
(\mathrm{ft} / \mathrm{s})\end{array}$ \\
\hline 3 & $1220^{a}$ & 8 & $1268^{a}$ \\
\hline 4 & 1250 & 10 & $1261^{a}$ \\
\hline 5 & 1238 & 12 & $1240^{a}$ \\
\hline 6 & 1261 & 14 & 1242 \\
\hline 7 & $1232^{a}$ & 16 & 1327 \\
\hline 9 & $1248^{a}$ & 17 & 1224 \\
\hline 11 & 1232 & 18 & $1264^{a}$ \\
\hline 13 & $1224^{a}$ & 19 & $1252^{\mathrm{a}}$ \\
\hline 15 & 1198 & & \\
\hline 20 & $1181^{a}$ & & \\
\hline
\end{tabular}

$a_{\text {Used to calculate }} v_{50}$ ballistic limit. 
Sample 25 (unused); tested dry

\begin{tabular}{|c|c|c|c|}
\hline \multicolumn{4}{|c|}{ Front } \\
\hline $\begin{array}{r}P a \\
\text { pene }\end{array}$ & $\begin{array}{l}\text { etial } \\
\text { eration }\end{array}$ & $\begin{array}{l}\text { Com } \\
\text { pene }\end{array}$ & $\begin{array}{l}\text { olete } \\
\text { tration }\end{array}$ \\
\hline $\begin{array}{l}\text { Round } \\
\text { no. }\end{array}$ & $\begin{array}{c}\text { Average } \\
\text { velocity } \\
(\mathrm{ft} / \mathrm{s})\end{array}$ & $\begin{array}{l}\text { Round } \\
\text { no. }\end{array}$ & $\begin{array}{c}\text { Average } \\
\text { velocity } \\
\text { (ft/s) }\end{array}$ \\
\hline $\begin{array}{r}4 \\
7 \\
11 \\
15 \\
18\end{array}$ & $\begin{array}{l}1212^{a} \\
1208^{a} \\
1245^{a} \\
1170^{a} \\
1122^{a}\end{array}$ & $\begin{array}{r}3 \\
5 \\
6 \\
8 \\
9 \\
10 \\
12 \\
13 \\
14 \\
16 \\
17\end{array}$ & $\begin{array}{l}1212^{a} \\
1188 \\
1242 \\
1304 \\
1261 \\
1250 \\
1202^{a} \\
1190^{a} \\
1238^{a} \\
1277 \\
1174 a\end{array}$ \\
\hline
\end{tabular}

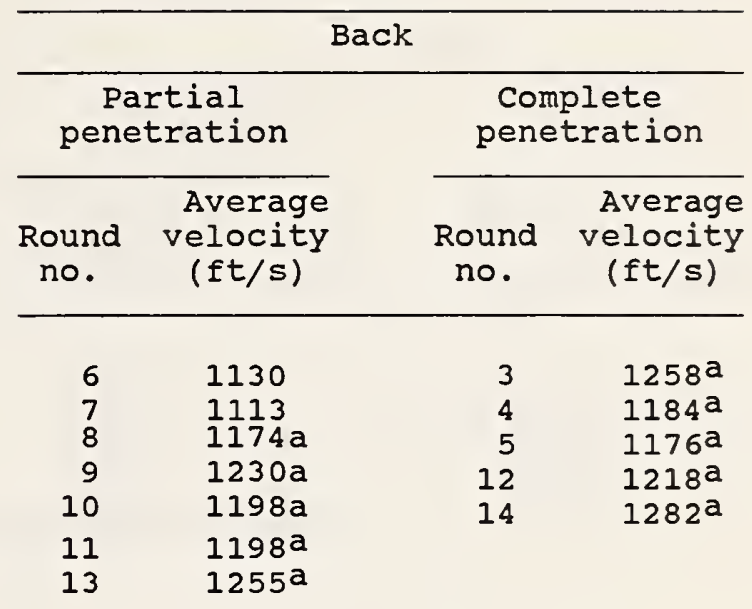

Sample 26/27 (unused); tested wet

\begin{tabular}{|c|c|c|c|}
\hline & \multicolumn{3}{|c|}{ Front (27) } \\
\hline \multicolumn{2}{|c|}{$\begin{array}{c}\text { Partial } \\
\text { penetration }\end{array}$} & \multicolumn{2}{|c|}{$\begin{array}{l}\text { Complete } \\
\text { penetration }\end{array}$} \\
\hline $\begin{array}{c}\text { Round } \\
\text { no. }\end{array}$ & $\begin{array}{c}\text { Average } \\
\text { velocity } \\
\text { (ft/s) }\end{array}$ & $\begin{array}{l}\text { Round } \\
\text { no. }\end{array}$ & $\begin{array}{c}\text { Average } \\
\text { velocity } \\
\text { (ft/s) }\end{array}$ \\
\hline 5 & $1174 \mathrm{a}$ & 4 & $1285^{a}$ \\
\hline 6 & $1238^{a}$ & 7 & $1268^{a}$ \\
\hline 11 & $1172^{a}$ & 8 & $1186^{a}$ \\
\hline 12 & $1186^{a}$ & 9 & $1248^{a}$ \\
\hline 13 & $1188^{a}$ & 10 & $1160^{a}$ \\
\hline
\end{tabular}

\begin{tabular}{|c|c|c|c|}
\hline \multicolumn{4}{|c|}{ Back (26) } \\
\hline \multicolumn{2}{|c|}{$\begin{array}{l}\text { Partial } \\
\text { penetration }\end{array}$} & \multicolumn{2}{|c|}{$\begin{array}{l}\text { Complete } \\
\text { penetration }\end{array}$} \\
\hline $\begin{array}{l}\text { Round } \\
\text { no. }\end{array}$ & $\begin{array}{c}\text { Average } \\
\text { velocity } \\
\text { (ft/s) }\end{array}$ & $\begin{array}{l}\text { Round } \\
\text { no. }\end{array}$ & $\begin{array}{c}\text { Average } \\
\text { velocity } \\
\text { (ft/s) }\end{array}$ \\
\hline 3 & $1222^{a}$ & 5 & $1313^{a}$ \\
\hline 4 & $1252^{a}$ & 7 & $1285^{a}$ \\
\hline 6 & $1282^{a}$ & 8 & $1238^{a}$ \\
\hline 9 & $1242^{\mathrm{a}}$ & 10 & 1222 \\
\hline 11 & $1288^{a}$ & 13 & $1248 a$ \\
\hline & & 14 & $1232^{a}$ \\
\hline
\end{tabular}

$a_{\text {Used to calculate }} \mathrm{v}_{50}$ ballistic limit. 
Sample 32 (moderate wear); tested dry

\begin{tabular}{|c|c|c|c|}
\hline \multicolumn{4}{|c|}{ Front } \\
\hline \multicolumn{2}{|c|}{$\begin{array}{c}\text { Partial } \\
\text { penetration }\end{array}$} & \multicolumn{2}{|c|}{$\begin{array}{l}\text { Complete } \\
\text { penetration }\end{array}$} \\
\hline $\begin{array}{l}\text { Round } \\
\text { no. }\end{array}$ & $\begin{array}{c}\text { Average } \\
\text { velocity } \\
(\mathrm{ft} / \mathrm{s})\end{array}$ & $\begin{array}{l}\text { Round } \\
\text { no. }\end{array}$ & $\begin{array}{c}\text { Average } \\
\text { velocity } \\
\text { (ft/s) }\end{array}$ \\
\hline 4 & $1200^{a}$ & 3 & $1266^{\mathrm{a}}$ \\
\hline 5 & $1268^{a}$ & 6 & $1250^{a}$ \\
\hline 7 & $1232^{a}$ & 8 & $1261^{a}$ \\
\hline 11 & $1215 a$ & 9 & $1215 a$ \\
\hline 12 & $1210^{a}$ & 10 & $1258 \mathrm{a}$ \\
\hline
\end{tabular}

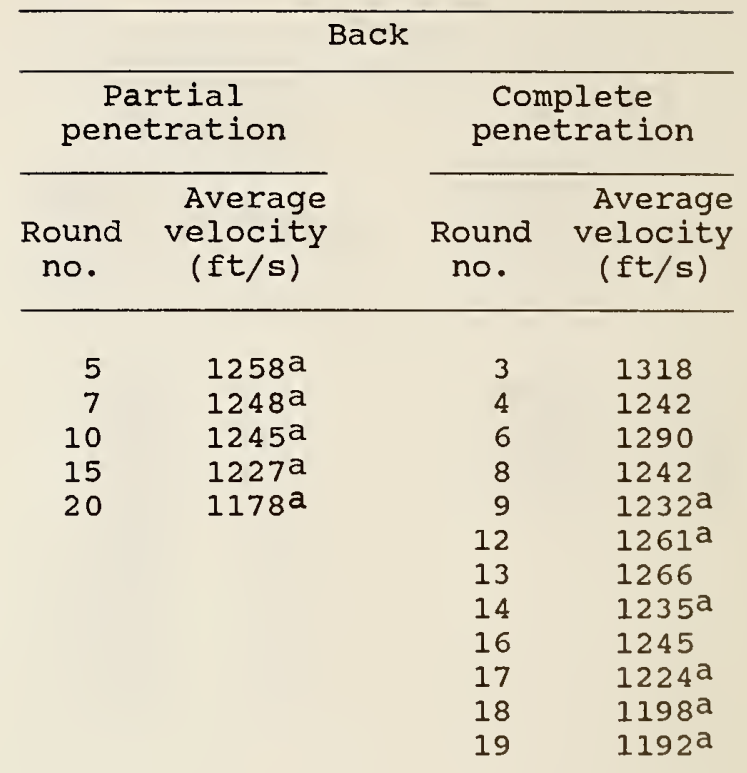

used to calculate $v_{50}$ ballistic limit. 
NBS.114A (REV. 2-8C)

U.S. DEPT. OF COMM.

BIBLIOGRAPHIC DATA

SHEET (See in structions)

1. PUBLICATION OR

REPORT NO.

NBSIR 86-3444

2. Performing Organ. Report No. 3. Publication Date

September 1986

4. TITLE AND SUBTITLE

Ballistic Tests of Used Soft Body Armor

5. AUTHOR(S)

Daniel E. Frank; 702

6. PERFORMING ORGANIZATION (If joint or other than NBS, see instructions)

7. Contract/Grant No.

NATIONAL BUREAU OF STANDARDS

DEPARTMENT OF COMMERCE

WASHINGTON, D.C. 20234

8. Type of Report \& Period Covered

9. SPONSORING ORGANIZATION NAME AND COMPLETE ADDRESS (Street, City, Stote, ZIP)

National Institute of Justice

U.S. Department of Justice

Washington, DC 20531

10. SUPPLEMENTARY NOTES

Document describes a computer program; SF-185, FIPS Software Summary, is attached.

11. ABSTRACT (A 200-word or less foctual summary of most significant informotion. If document includes a significant bibliography or literature survey, mention it here)

A sample of 24 ballistic resistant undergarments (soft body armor) from a production lot of 1500 originally distributed to 15 police departments throughout the United States in 1975 for issue to officers as part of a Law Enforcement Assistance Administration demonstration project, was tested for $V_{5}$ ballistic limit. The program was a joint effort of the U.S. Department of Justice National Institute of Justice and the National Research Council of Canada Public Safety project office. Tests of ballistic limit were conducted on virgin armor that were never issued, and armor showing evidence of light, moderate, and heavy wear both dry and while wet. The results show that armor does not lose ballistic efficiency as a consequence of age.

12. KEY WORDS (Six to twelve entries; alphabetical order; capitalize only proper names; and separate key words by semicolons) ballistic limit; ballistic-resistant body armor; ballistic testing; body armor; Kevlar; soft body armor

13. AVAILABILITY

[X] Unlimited

$\square$ For Official Distribution. Do Not Release to NTIS

[ Order From Superintendent of Documents, U.S. Government Printing Office, Washington. D.C. 20402.

[X] Order From National Technical Information Service (NTIS), Springfield, VA. 2216I
14. NO. OF

PRINTED PAGES

44

15. Price 


